\title{
THE $A$-HYPERGEOMETRIC SYSTEM ASSOCIATED WITH A MONOMIAL CURVE
}

\author{
EDUARDO CATTANI, CARLOS D'ANDREA, AND ALICIA DICKENSTEIN
}

Introduction. In this paper we make a detailed analysis of the $\mathscr{A}$-hypergeometric system (or GKZ system) associated with a monomial curve and integral, hence resonant, exponents. We describe all rational solutions and show in Theorem 1.10 that they are, in fact, Laurent polynomials. We also show that for any exponent there are at most two linearly independent Laurent solutions and that the upper bound is reached if and only if the curve is not arithmetically Cohen-Macaulay. We then construct, for all integral parameters, a basis of local solutions in terms of the roots of the generic univariate polynomial $(0.5)$ associated with $\mathscr{A}$. We also determine in Theorem 3.7 the holonomic rank $r(\alpha)$ for all $\alpha \in \mathbb{Z}^{2}$ and show that $d \leq r(\alpha) \leq d+1$, where $d$ is the degree of the curve. Moreover, the value $d+1$ is attained only for those exponents $\alpha$ for which there are two linearly independent rational solutions, and, therefore, $r(\alpha)=d$ for all $\alpha$ if and only if the curve is arithmetically Cohen-Macaulay.

In order to place these results in their appropriate context, we recall the definition of the $\mathscr{A}$-hypergeometric systems. These were introduced in a series of papers in the mid-1980s by the Gel'fand school, particularly Gel'fand, Kapranov, and Zelevinsky (see [7] and [9] and the references therein). Let $\mathscr{A}=\left\{v_{1}, \ldots, v_{r}\right\} \subset \mathbb{Z}^{n+1}$ be a finite subset that spans the lattice $\mathbb{Z}^{n+1}$. Suppose, moreover, that there exists a vector $\lambda=$ $\left(\lambda_{0}, \ldots, \lambda_{n}\right) \in \mathbb{Q}^{n+1}$ such that $\left\langle\lambda, v_{j}\right\rangle=1$ for all $j=1, \ldots, r$, that is, the set $\mathscr{A}$ lies in a rational hyperplane. Let $\mathscr{A}$ also denote the $(n+1) \times r$ matrix whose columns are the vectors $v_{j}$. Let $\mathscr{L} \subset \mathbb{Z}^{r}$ be the sublattice of elements $v \in \mathbb{Z}^{r}$ such that $\mathscr{A} \cdot v=0$. Given $\alpha \in \mathbb{C}^{n+1}$, the A-hypergeometric system with exponent (or parameter) $\alpha$ is

$$
\begin{aligned}
\mathscr{D}_{v} \varphi & =0, \quad v \in \mathscr{L} ; \\
\sum_{j=1}^{r} a_{i j} x_{j} \frac{\partial \varphi}{\partial x_{j}} & =\alpha_{i} \varphi, \quad i=1, \ldots, n+1,
\end{aligned}
$$

where $\mathscr{A}=\left(a_{i j}\right)$ and $\mathscr{D}_{v}$ is the differential operator in $\mathbb{C}^{r}$ :

Received 29 December 1997. Revision received 1 September 1998.

1991 Mathematics Subject Classification. Primary 33C70; Secondary 14M05, 33D20.

Cattani's work supported by National Science Foundation grant number DMS-9406462.

D'Andrea's work supported by doctoral fellowship from Fondo para el Mejoramiento de la Calidad Educativa, Argentina.

Dickenstein's work supported by Proyecto UBACYT TX94, Secretaría de Ciencia y Técnica, Universidad de Buenos Aires, Argentina, and Consejo Nacional de Investigaciones Científicas y Técnicas, Argentina. 


$$
\mathscr{D}_{v}:=\prod_{v_{j}>0}\left(\frac{\partial}{\partial x_{j}}\right)^{v_{j}}-\prod_{v_{k}<0}\left(\frac{\partial}{\partial x_{k}}\right)^{-v_{k}} \text {. }
$$

The $\mathscr{A}$-hypergeometric system is holonomic (with regular singularities) and, consequently, the number of linearly independent solutions at a generic point is finite (see [7]). Let $r(\alpha)$ denote the holonomic rank of the system, that is, the dimension of the space of local solutions at a generic point in $\mathbb{C}^{r}$. If we drop the assumption that $\mathscr{A}$ lies in a hyperplane, then the regular singularities property is lost, but, as Adolphson [2] has shown, the system remains holonomic. The singular locus is described by the zeroes of the principal $\mathscr{A}$-determinant (see [11]). We set $R:=\mathbb{C}\left[\xi_{1}, \ldots, \xi_{r}\right] / \mathscr{I}_{\mathscr{A}}$, where $I_{\mathscr{A}}$ is the toric ideal

$$
I_{\mathscr{A}}:=\left\langle\xi^{u}-\xi^{v}: u, v \in \mathbb{N}^{r} ; \mathscr{A} \cdot u=\mathscr{A} \cdot v\right\rangle .
$$

When $n=1$, we can assume without loss of generality that

$$
\mathscr{A}=\left(\begin{array}{ccccc}
1 & 1 & \cdots & 1 & 1 \\
0 & k_{1} & \cdots & k_{m} & d
\end{array}\right)
$$

where $0<k_{1}<\cdots<k_{m}<d$. Note that the condition that the columns of $\mathscr{A}$ generate the lattice $\mathbb{Z}^{2}$ is equivalent to $\operatorname{gcd}\left(k_{1}, \ldots, k_{m}, d\right)=1$. The homogeneous ideal $\Phi_{\mathscr{A}}$ defines a monomial curve $X_{\mathscr{A}} \subset \mathbb{P}^{m+1}$ of degree $d$ whose homogeneous coordinate ring is $R . X_{\mathscr{A}}$ is normal if and only if $d=m+1$. Recall that $X_{\mathscr{A}}$ is said to be arithmetically Cohen-Macaulay if and only if the ring $R$ is Cohen-Macaulay.

The system associated with (0.4) admits very interesting solutions. Let

$$
f(x ; t):=x_{0}+x_{k_{1}} t^{k_{1}}+\cdots+x_{k_{m}} t^{k_{m}}+x_{d} t^{d}
$$

denote the generic polynomial with exponents $0, k_{1}, \ldots, k_{m}, d$. It is not hard to see that the powers $\rho^{s}(x), s \in \mathbb{Z}$, of the roots of $f(x ; t)$, viewed as functions of the coefficients, are algebraic solutions of the $\mathscr{A}$-hypergeometric system with exponent $(0,-s)$. This fact was observed by Mayr [17], who constructed series expansions for these functions. These have more recently been refined by Sturmfels [22]. The total sum

$$
p_{s}(x):=\rho_{1}^{s}(x)+\cdots+\rho_{d}^{s}(x)
$$

is then a rational solution with the same exponent. Similarly, one can show that the local residues

$$
\operatorname{Res}_{\rho(x)}\left(\frac{t^{b}}{f^{a}(x ; t)} \frac{d t}{t}\right), \quad a, b \in \mathbb{Z} ; a \geq 1
$$

give algebraic solutions with exponent $(-a,-b)$ and, again, the total sum of residues is a rational solution. 
In $\S 1$ we describe explicitly all rational solutions of the $\mathscr{A}$-hypergeometric system associated with a monomial curve. Since for $\mathscr{A}$ as in (0.4) the principal $\mathscr{A}$-determinant factors into powers of $x_{0}, x_{d}$, and the discriminant $\Delta(f)$, we know a priori what the possible denominators of a rational solution may be. However, we show in Theorem 1.10 that there are no rational solutions with a denominator that involves $\Delta(f)$, and, therefore, every rational solution must be a Laurent polynomial. This is a somewhat surprising result that is peculiar to the case $n=1$ (see Example 1.11). One may give explicit formulas for these Laurent polynomials in terms of hypergeometric polynomials in fewer variables. When applied to the sum of powers of roots, one recovers the classical Girard formulas. One also obtains similar expressions for total residues in terms of hypergeometric polynomials.

We show that for any $\alpha \in \mathbb{Z}^{2}$ the dimension of the space of rational $\mathscr{A}$-hypergeometric functions with parameter $\alpha$ is at most 2. Moreover, the value 2 may be reached for only finitely many values of $\alpha$, and this happens if and only if the ring $R$ is not Cohen-Macaulay.

In $\S 2$ we exhibit a family of algebraic $\mathscr{A}$-hypergeometric functions defined in terms of the roots of the polynomial $f(x ; t)$. These are the building blocks for the construction, in $\S 3$, of local bases of solutions and the determination of the holonomic rank for all integral exponents. It becomes necessary to consider four possibilities for the exponent $\alpha$. These cases admit combinatorial descriptions (see (1.9)) and correspond to the existence of a polynomial solution, a 1-dimensional space of rational-nonpolynomial-solutions, a 2-dimensional space of rational solutions, or no rational solution for the given exponent. For $u \in \mathbb{N}^{m+2}$, the derivative $D_{u}$ maps $\mathscr{A}$-hypergeometric functions to $\mathscr{A}$-hypergeometric functions while changing the exponent from $\alpha$ to $\alpha-\mathscr{A} \cdot u$. A careful analysis of the kernel and image of this operator together with Corollary 5.20 of [2] leads to the determination of the holonomic rank for all values of $\alpha$. We show, in particular, that

$$
d \leq r(\alpha) \leq d+1
$$

and that $r(\alpha)=d+1$ exactly for those parameters $\alpha \in \mathbb{Z}^{2}$ for which the dimension of the space of rational solutions is 2 . Hence, $r(\alpha)=d$ for all $\alpha \in \mathbb{Z}^{2}$ if and only if the curve $X_{\mathscr{A}}$ is arithmetically Cohen-Macaulay.

These results allow us to clarify the relationship between the holonomic rank and $\operatorname{vol}(P)$, the normalized volume of the convex hull $P \subset \mathbb{R}^{n+1}$ of $\mathscr{A}$ and the origin (which equals the degree $d$ in the case of curves). It was originally claimed in [9, Theorem 2] that $r(\alpha)=\operatorname{vol}(P)$ in all cases, but later it was pointed out by Adolphson that, for resonant exponents, the proof required the assumption that the ring $R$ be Cohen-Macaulay (see [10]). In [2, Cor. 5.20], Adolphson showed that $r(\alpha)=\operatorname{vol}(P)$, for $\alpha$ a semi-nonresonant exponent, without any additional assumptions on $R$. The first explicit example where the equality fails is given in [23] and described in Example 1.8.i). In [19], Saito, Sturmfels, and Takayama prove (0.8) using Gröbner deformation methods and show that the inequality $r(\alpha) \geq \operatorname{vol}(P)$ 
holds without restrictions on $n$.

Very little seems to be known about the problem of finding rational solutions of differential equations beyond the case of linear differential operators in one variable. In this case, Singer [20] has shown that one can determine in a finite number of steps whether a given equation has a rational solution and find a basis for the space of such solutions. Abramov and Kvasenko [1] have further studied the problem of effectively finding rational solutions for such operators. In our case one could, in principle, use noncommutative elimination to obtain linear operators in one variable with coefficients that depend rationally on the other variables and apply Singer's decision procedure to characterize the rational solutions. This can be done in small examples using noncommutative Gröbner-bases packages such as KAN (see [24]), but we have not been able to obtain any general results in this manner.

Gel'fand, Zelevinsky, and Kapranov have constructed series solutions for (0.1)(0.2), associated with regular triangulations of the polytope $P$. When the exponent $\alpha$ is nonresonant for the triangulation, it is possible to obtain in this manner $\operatorname{vol}(P)$ many independent solutions. There are, however, very interesting cases in which the exponents are integral and, therefore, automatically resonant. For example, it has been observed by Batyrev in [4] that the period integrals of Calabi-Yau hypersurfaces in toric varieties satisfy an $\mathcal{A}$-hypergeometric system with exponents $\alpha=(-1,0, \ldots, 0)$. In this case, series solutions have been obtained by Hosono, Lian, and Yau ([14], [15]; see also [3], [5], and [13]). Very recently, Stienstra [21] has generalized the $\Gamma$-series construction of Gel'fand, Zelevinsky, and Kapranov to obtain series solutions in the case of resonant exponents under a maximal-degeneracy assumption. In particular, if $\alpha=0$ and $P$ admits a unimodular triangulation-which implies that $R$ is CohenMacaulay - all solutions of (0.1)-(0.2) may be obtained in this manner. This method also yields all solutions of interest in the context of toric mirror symmetry.

A key result, in the curve case, is Theorem 1.9, which asserts that for $m \geq 1$ there are no rational solutions with integral exponents in the Euler-Jacobi cone. This corresponds to the classical vanishing theorem for the total sum of residues, a statement that has a generalization as the Euler-Jacobi theorem (see [16] for the most general form of this result). It is interesting to note then that Euler-Jacobi vanishing is a consequence of the fact that residues satisfy the $\mathscr{A}$-hypergeometric system. We also point out that while the characterization of Laurent solutions follows from formal arguments, the proof of the Euler-Jacobi vanishing involves transcendental methods.

Acknowledgments. We are grateful to B. Sturmfels for many helpful comments and, particularly, for the statement and proof of Proposition 1.6. We also thank A. Zelevinsky for useful suggestions and the two referees for their careful reading of the manuscript and very thoughtful suggestions for improvement.

1. Rational solutions. The polynomial solutions of a general $\mathscr{A}$-hypergeometric system admit a very simple description. Given $\alpha \in \mathbb{Z}^{r}$, we define the hypergeometric polynomial 


$$
\Phi^{\mathscr{A}}(\alpha ; x):=\sum_{\substack{u \in \mathbb{N}^{r} \\ \mathscr{A} \cdot u=\alpha}} \frac{x^{u}}{u !}=\sum_{\substack{u \in \mathbb{N}^{r} \\ \mathbb{A} \cdot u=\alpha}} \frac{x_{1}^{u_{1}} x_{2}^{u_{2}} \cdots x_{r}^{u_{r}}}{u_{1} ! u_{2} ! \cdots u_{r} !} .
$$

As usual, we set $\Phi^{\mathscr{A}}(\alpha ; x):=0$ if $\alpha \notin \mathscr{A} \cdot \mathbb{N}^{r}$. The following result, the verification of which is left to the reader, is Proposition 2.1 in [18].

Proposition 1.1. $\Phi^{A}(\alpha ; x)$ is the unique, up-to-scaling, polynomial solution of the A-hypergeometric system with exponent $\alpha$. Moreover, for any $u \in \mathbb{N}^{r}$,

$$
D_{u}\left(\Phi^{\mathscr{A}}(\alpha ; x)\right)=\Phi^{\mathscr{A}}(\alpha-\mathscr{A} \cdot u ; x)
$$

where $D_{u}$ stands for the partial derivative $\partial^{|u|} / \partial x^{u}$.

The purpose of this section is to describe the rational solutions of the $\mathscr{A}$-hypergeometric system associated with a matrix $\mathscr{A}$ as in (0.4). Note that for $m=0$, the system restricts to the homogeneity equations (0.2). Therefore, we may assume throughout that $m \geq 1$. To simplify our notation, we index all $(m+2)$-tuples by $0, k_{1}, \ldots, k_{m}, d$. Let $e_{0}, e_{k_{1}}, \ldots, e_{d}$ denote the standard basis of $\mathbb{Z}^{m+2}$. For $i=1, \ldots, m$ we have

$$
\omega_{k_{i}}:=\left(d-k_{i}\right) e_{0}-d e_{k_{i}}+k_{i} e_{d} \in \mathscr{L} .
$$

The following observation will be useful in the sequel.

Proposition 1.2. Suppose $\varphi$ is a local holomorphic solution of (0.1)-(0.2), which is polynomial with respect to any of the variables $x_{0}, x_{k_{1}}, \ldots, x_{d}$. Then $\varphi$ is a Laurent polynomial.

Proof. Since $\varphi$ satisfies the equations (0.1), it follows from (1.3) that for all $\ell \in \mathbb{N}$,

$$
D_{k_{i}}^{\ell d} \varphi=D_{0}^{\ell\left(d-k_{i}\right)} D_{d}^{\ell k_{i}} \varphi
$$

and, consequently, if $\varphi$ is polynomial in any of the variables, it must be so in all of the variables $x_{k_{i}}, i=1, \ldots, m$, and we may write

$$
\varphi(x)=\sum_{u} \varphi_{u}\left(x_{0}, x_{d}\right) \check{x}^{u}, \quad \check{x}=\left(x_{k_{1}}, \ldots, x_{k_{m}}\right),
$$

where $u$ varies in a finite subset of $\mathbb{N}^{m}$, and each $\varphi_{u}\left(x_{0}, x_{d}\right)$ is homogeneous in each variable with respective degrees $\beta_{0}, \beta_{d}$ satisfying $d \beta_{d} \in \mathbb{Z}, \beta_{0}+\beta_{d} \in \mathbb{Z}$. Thus, $\varphi_{u}\left(x_{0}, x_{d}\right)=c_{u} x_{0}^{\beta_{0}} x_{d}^{\beta_{d}}, c_{u} \in \mathbb{C}$. But, because of (1.4), for $r, s \in \mathbb{N}$ sufficiently large, $D_{0}^{r} D_{d}^{s} \varphi=0$. Consequently, one, and therefore both, of $\beta_{0}, \beta_{d}$ must be an integer. Hence, $\varphi$ is a Laurent polynomial.

Note that in the 1-dimensional case, an $\mathscr{A}$-hypergeometric Laurent polynomial may not contain a nonzero term of the form $c_{u} x^{u}$ with $u_{k_{i}}<0$. This follows from the fact 
that the singular locus $\Sigma$ of the hypergeometric system is given by the zeroes of the principal $\mathscr{A}$-determinant, that is,

$$
\Sigma=\left\{x_{0}=0\right\} \cup\left\{x_{d}=0\right\} \cup\{\Delta(f)=0\},
$$

where $\Delta(f)$ is the discriminant of the generic polynomial (0.5). Alternatively, if a solution contains a nonzero term $c_{u} x^{u}$, with $u_{k_{i}}<0$, being in the kernel of the differential operator $D_{k_{i}}^{d}-D_{0}^{d-k_{i}} D_{d}^{k_{i}}$, it must also contain nontrivial terms of the form $c_{v} x^{v}$, with $v_{k_{i}}=u_{k_{i}}-j d$ for all positive integers $j$, and this is clearly impossible. A similar argument shows that a Laurent solution may not contain terms of the form $c_{u} x^{u}$, with both $u_{0}<0$ and $u_{d}<0$.

Thus, any Laurent solution must be of the form

$$
L_{0}(x)+L_{d}(x)
$$

where $L_{0}(x)$ has as denominators only powers of $x_{0}$, and $L_{d}(x)$ has as denominators only powers of $x_{d}$. We note that the study of $L_{0}(x)$ and $L_{d}(x)$ is completely symmetric. Indeed, let $\ell_{j}=d-k_{m-j+1}, j=1, \ldots, m$,

$$
\hat{A}=\left(\begin{array}{ccccc}
1 & 1 & \cdots & 1 & 1 \\
0 & \ell_{1} & \cdots & \ell_{m} & d
\end{array}\right),
$$

and suppose $R(x)$ is a Laurent solution of the $\mathscr{A}$-hypergeometric system and exponent $\alpha=\left(\alpha_{1}, \alpha_{2}\right)$. (Although $\alpha$ should be viewed as a column vector, we always write exponents as row vectors for simplicity of notation.) Then the function $\hat{R}\left(y_{0}, y_{\ell_{1}}, \ldots, y_{\ell_{m}}, y_{d}\right)$ obtained from $R$ by the substitution

$$
x_{0} \mapsto y_{d}, \quad x_{k_{j}} \mapsto y_{\ell_{j}}, \quad x_{d} \mapsto y_{0},
$$

is a solution of the $\hat{A}$-hypergeometric system and exponents $\hat{\alpha}=\left(\alpha_{1}, d \alpha_{1}-\alpha_{2}\right)$.

Lemma 1.3. For $\alpha \in \mathscr{A} \cdot \mathbb{N}^{m+2}$, the only Laurent solutions of the $\mathscr{A}$-hypergeometric system are the constant multiples of the hypergeometric polynomial (1.1).

Proof. Suppose there is a Laurent solution $L(x)$ of exponent $\alpha$ containing a term of the form $x^{u} / x_{d}^{r}$, with $u \in \mathbb{N}^{m+2}, u_{d}=0$, and $r>0$ (we always assume that monomials are written in reduced form). Then $\mathscr{A} \cdot\left(u-r e_{d}\right)=\alpha$. Let $v \in \mathbb{N}^{m+2}$ be such that $\mathscr{A} \cdot v=\alpha$; then $\mathscr{A} \cdot u=\mathscr{A} \cdot\left(v+r e_{d}\right)$, and the operator $D_{u}-D_{d}^{r} D_{v}$, being in the hypergeometric system, must vanish on $L$. This means that $L$ must also contain a term $x^{w}$ whose derivative $D_{d}^{r} D_{v}\left(x^{w}\right)$ is a nonzero multiple of $1 / x_{d}^{r}$. Since $v_{d} \geq 0$, this is clearly impossible. Arguing by symmetry, we see that there cannot be a solution containing a term of the form $x^{u} / x_{0}^{r}$, with $u_{0}=0$ and $r>0$.

We denote by $\mathscr{B}$ the matrix

$$
\mathscr{B}=\left(\begin{array}{cccc}
1 & 1 & \cdots & 1 \\
0 & k_{1} & \cdots & k_{m}
\end{array}\right)
$$


and by $x^{\prime}$ the vector consisting of the first $m+1$ variables $\left(x_{0}, x_{k_{1}}, \ldots, x_{k_{m}}\right)$. Similarly, let $\mathscr{b}$ be the matrix

$$
\mathscr{C}=\left(\begin{array}{cccc}
1 & \cdots & 1 & 1 \\
k_{1} & \cdots & k_{m} & d
\end{array}\right)
$$

and $\tilde{x}$ the vector $\left(x_{k_{1}}, \ldots, x_{k_{m}}, x_{d}\right)$.

Given $\alpha \in \mathbb{Z}^{2}$ we define

$$
\Psi_{d}^{A}(\alpha ; x):=\sum_{r \geq 1}(-1)^{r}(r-1) ! \frac{\Phi^{\mathscr{P}}\left(\alpha^{\prime}(r) ; x^{\prime}\right)}{x_{d}^{r}},
$$

where $\alpha^{\prime}(r)=\alpha+r(1, d)$, and

$$
\Psi_{0}^{\unlhd}(\alpha ; x):=\sum_{r \geq 1}(-1)^{r}(r-1) ! \frac{\Phi^{\mathscr{C}}(\tilde{\alpha}(r) ; \tilde{x})}{x_{0}^{r}},
$$

where $\tilde{\alpha}(r)=\alpha+r(1,0)$.

Note that both sums are finite. This follows from the fact that $\Phi^{\mathscr{B}}\left(\alpha^{\prime}(r) ; x^{\prime}\right)=0$ unless $\alpha^{\prime}(r) \in \mathscr{B} \cdot \mathbb{N}^{m+1}$; but then $\alpha_{2}+d r \leq k_{m}\left(\alpha_{1}+r\right)$, and, therefore, $\left(d-k_{m}\right) r \leq$ $k_{m} \alpha_{1}-\alpha_{2}$. This means that the possible values of $r$ in (1.7) are bounded by $\left(k_{m} \alpha_{1}-\right.$ $\left.\alpha_{2}\right) /\left(d-k_{m}\right)$. The statement for (1.8) follows by symmetry. In fact, we should observe that the change of variables (1.6) transforms $\Psi_{d}^{A}(\alpha ; x)$ into $\Psi_{0}^{\hat{A}}(\hat{\alpha} ; x)$.

The following subsets of $\mathbb{Z}^{2}$ play an important role in the description of $\mathscr{A}$-hypergeometric functions:

$$
\begin{aligned}
& I(\mathscr{A}):=\mathscr{A} \cdot \mathbb{N}^{m+2} \\
& F_{0}(\mathscr{A}):=\mathscr{C} \cdot \mathbb{N}^{m+1}-\mathbb{N}(1,0) ; \quad E_{0}(\mathscr{A}):=F_{0}(\mathscr{A}) \backslash I(\mathscr{A}) ; \\
& F_{d}(\mathscr{A}):=\mathscr{B} \cdot \mathbb{N}^{m+1}-\mathbb{N}(1, d) ; \quad E_{d}(\mathscr{A}):=F_{d}(\mathscr{A}) \backslash I(\mathscr{A}) ; \\
& E(\mathscr{A}):=E_{0}(\mathscr{A}) \cap E_{d}(\mathscr{A}) ; \quad J(\mathscr{A}):=\mathbb{Z}^{2} \backslash\left(I(\mathscr{A}) \cup F_{0}(\mathscr{A}) \cup F_{d}(\mathscr{A})\right) .
\end{aligned}
$$

Note that via the change of variables (1.6), and denoting for $\alpha=\left(\alpha_{1}, \alpha_{2}\right) \in \mathbb{Z}^{2}$, $\hat{\alpha}=\left(\alpha_{1}, d \alpha_{1}-\alpha_{2}\right)$, we have for $\hat{\mathscr{A}}$ as in (1.5):

$$
I(\hat{\mathscr{A}})=\widehat{I(\mathscr{A})} ; \quad F_{0}(\hat{\mathscr{A}})=\widehat{F_{d}(\mathscr{A})} ; \quad F_{d}(\hat{\mathscr{A}})=\widehat{F_{0}(\mathscr{A})} .
$$

It is clear from the definitions (1.7) and (1.8) that $\Psi_{0}^{\mathscr{A}}(\alpha ; x) \neq 0$ if and only if $\alpha \in F_{0}(\mathscr{A})$ and, similarly, $\Psi_{d}^{\mathscr{A}}(\alpha ; x) \neq 0$ if and only if $\alpha \in F_{d}(\mathscr{A})$. In particular,

$$
\Psi_{d}^{\unlhd}(\alpha ; x)=0, \quad \text { if } d \alpha_{1}<\alpha_{2},
$$

and

$$
\Psi_{0}^{A}(\alpha ; x)=0, \quad \text { if } \alpha_{2}<0 .
$$

On the other hand, the importance of the sets $E_{0}(\mathscr{A})$ and $E_{d}(\mathscr{A})$ stems from the fact 
that according to Lemma 1.3 , it is only for $\alpha \in E_{0}(\mathscr{A})$ (respectively, $\alpha \in E_{d}(\mathscr{A})$ ) that the Laurent polynomial $\Psi_{0}^{A}(\alpha ; x)$ (respectively, $\Psi_{d}^{A A}(\alpha ; x)$ ) may be-and as the following result shows is- $\mathscr{A}$-hypergeometric. Note also that there are no $\mathscr{A}$ hypergeometric Laurent polynomials with exponent $\alpha \in J(\mathscr{A})$, and it is a consequence of Theorem 1.10 that there are no rational $\mathscr{A}$-hypergeometric functions with exponent $\alpha \in J(\mathscr{A})$.

TheOREM 1.4. Let $\mathscr{A}$ be as in (0.4) and $\alpha \in \mathbb{Z}^{2} \backslash I(\mathscr{A})$. Then:

(i) $\Psi_{d}^{A A}(\alpha ; x)$ and $\Psi_{0}^{A A}(\alpha ; x)$ are solutions (possibly trivial) of the A-hypergeometric system with parameter $\alpha$;

(ii) for any $u \in \mathbb{N}^{m+2}$,

$$
D_{u}\left(\Psi_{d}^{A}(\alpha ; x)\right)=\Psi_{d}^{A}(\alpha-\mathscr{A} \cdot u ; x) ; \quad D_{u}\left(\Psi_{0}^{A}(\alpha ; x)\right)=\Psi_{0}^{\mathscr{A}}(\alpha-\mathscr{A} \cdot u ; x) ;
$$

(iii) the functions $\Psi_{d}^{A}(\alpha ; x)$ and $\Psi_{0}^{A}(\alpha ; x)$ span the space of Laurent solutions of the A-hypergeometric system with parameter $\alpha$.

Proof. Clearly, (i) is an immediate consequence of (ii) and, because of symmetry, it suffices to show (1.12) for $\Psi_{d}^{\unlhd}(\alpha ; x)$. Suppose $u \in \mathbb{N}^{m+2}$ is such that $u_{d}=0$; then

$$
\begin{aligned}
D_{u}\left(\Psi_{d}^{\mathscr{A}}(\alpha ; x)\right) & =\sum_{r \geq 1}(-1)^{r}(r-1) ! \frac{D_{u}\left(\Phi^{\mathscr{B}}\left(\alpha^{\prime}(r) ; x^{\prime}\right)\right)}{x_{d}^{r}} \\
& =\sum_{r \geq 1}(-1)^{r}(r-1) ! \frac{\Phi^{\mathscr{B}}\left(\alpha^{\prime}(r)-\mathscr{B} \cdot u ; x^{\prime}\right)}{x_{d}^{r}} \\
& =\Psi_{d}^{\mathscr{A}}(\alpha-\mathscr{A} \cdot u ; x) .
\end{aligned}
$$

Thus, it remains to prove (1.12) for the partial derivative $D_{d}$. We have

$$
\begin{aligned}
D_{d} \Psi_{d}^{A}(\alpha ; x) & =\sum_{r \geq 1}(-1)^{r+1} r ! \frac{\Phi^{\mathscr{R}}\left(\alpha^{\prime}(r) ; x^{\prime}\right)}{x_{d}^{r+1}} \\
& =\sum_{r \geq 1}(-1)^{r+1} r ! \frac{\Phi^{\mathscr{P}}\left(\left(\alpha-\mathscr{A} \cdot e_{d}\right)^{\prime}(r+1) ; x^{\prime}\right)}{x_{d}^{r+1}} \\
& =\Psi_{d}^{A}\left(\alpha-\mathscr{A} \cdot e_{d} ; x\right) .
\end{aligned}
$$

The last equality follows, since $\alpha \notin \mathscr{A} \cdot \mathbb{N}^{m+2}$ implies that

$$
\Phi^{\mathscr{B}}\left(\left(\alpha-\mathscr{A} \cdot e_{d}\right)^{\prime}(1) ; x^{\prime}\right)=\Phi^{\mathscr{B}}\left(\alpha ; x^{\prime}\right)=0 .
$$

Suppose now that $L(x)$ is a Laurent solution with exponent $\alpha$, and write $L(x)=$ $L_{d}(x)+L_{0}(x)$. If we decompose further,

$$
L_{d}(x)=\sum_{r \geq 1} \frac{A_{r}\left(x^{\prime}\right)}{x_{d}^{r}},
$$


then the polynomials $A_{r}\left(x^{\prime}\right)$ must be solutions of the $\mathscr{B}$-hypergeometric system and exponent $\alpha^{\prime}(r)$. Thus, $A_{r}\left(x^{\prime}\right)=c_{r} \Phi^{\mathscr{P}}\left(\alpha^{\prime}(r) ; x^{\prime}\right)$. Assume now that for $r, s \geq 1, r \neq s$, we have $\alpha^{\prime}(r), \alpha^{\prime}(s) \in I(\mathscr{B})$, and let $v, w \in \mathbb{N}^{m+1}$ be such that $\mathscr{B} \cdot v=\alpha^{\prime}(r)$ and $\mathscr{B} \cdot w=\alpha^{\prime}(s)$. Then, since $\mathscr{A} \cdot\left(v+s e_{d}\right)=\mathscr{A} \cdot\left(w+r e_{d}\right)$, the operator

$$
\frac{\partial^{|v|+s}}{\partial x^{v} x_{d}^{s}}-\frac{\partial^{|w|+r}}{\partial x^{w} x_{d}^{r}}
$$

is in the hypergeometric system and must vanish on $L$. This means that

$$
\frac{(-1)^{s}(r+s-1) !}{(r-1) ! x_{d}^{r+s}} \frac{\partial^{|v|}\left(A_{r}\left(x^{\prime}\right)\right)}{\partial x^{v}}=\frac{(-1)^{r}(r+s-1) !}{(s-1) ! x_{d}^{r+s}} \frac{\partial^{|w|}\left(A_{s}\left(x^{\prime}\right)\right)}{\partial x^{w}},
$$

which implies that

$$
\frac{(-1)^{s}}{(r-1) !} c_{r}=\frac{(-1)^{r}}{(s-1) !} c_{s}
$$

and, consequently,

$$
c_{r}=c(-1)^{r}(r-1) ! .
$$

A symmetric argument shows that if the component $L_{0}(x)$ is nontrivial, then it must be a constant multiple of $\Psi_{0}^{A}(\alpha ; x)$, which proves part (iii).

We state for emphasis the following result.

Corollary 1.5. Let $\mathscr{A}$ be as in (0.4), and let $\mathscr{L}(\alpha)$ denote the vector space of $\mathscr{A}$ hypergeometric Laurent polynomials of exponent $\alpha$. Then $\operatorname{dim} \mathscr{L}(\alpha) \leq 2$. Moreover, $\operatorname{dim} \mathscr{L}(\alpha) \geq 1$ if and only if $\alpha \in I(\mathscr{A}) \cup E_{0}(\mathscr{A}) \cup E_{d}(\mathscr{A})$, and $\operatorname{dim} \mathscr{L}(\alpha)=2$ if and only if $\alpha \in E(\mathscr{A})$.

The following result was brought to our attention by Bernd Sturmfels.

Proposition 1.6. Given $\mathscr{A}$ as in $(0.4)$, the ring $R=\mathbb{C}\left[\xi_{0}, \ldots, \xi_{d}\right] / \mathscr{I}_{\mathscr{A}}$ is CohenMacaulay if and only if $E(\mathscr{A})=\emptyset$.

Proof. The ring $R$ is a particularly simple example of an affine semigroup ring whose properties have been extensively studied (see, for example, [6, Chap. 6], [12], and [25]). In fact, Proposition 1.6 is a special case of Theorem 2.6 in [12], which gives necessary and sufficient conditions for an affine semigroup ring that, like $R$ does, admits a system of monomial parameters. Their condition (ii) is easily seen to be equivalent, in our notation, to $E(\mathscr{A})=\emptyset$.

1.7. Remarks. (i) When the curve $X_{\mathscr{A}}$ is normal, that is, $d=m+1$, the sets defined in (1.9) have a very simple description: The image $I(\mathscr{A})$ coincides with the "cone" (properly speaking, semigroup)

$$
\mathscr{C}:=\left\{\alpha=\left(\alpha_{1}, \alpha_{2}\right) \in \mathbb{Z}^{2}: 0 \leq \alpha_{2} \leq d \alpha_{1}\right\},
$$


while $E_{0}(\mathscr{A})=\left\{\alpha \in \mathbb{Z}^{2}: \alpha_{2} \geq 0, d \alpha_{1}<\alpha_{2}\right\}$ and $E_{d}(\mathscr{A})=\left\{\alpha \in \mathbb{Z}^{2}: \alpha_{2}<0, d \alpha_{1} \geq\right.$ $\left.\alpha_{2}\right\}$. The complement $J(\mathscr{A})$ of these three sets, that is, the set of $\alpha \in \mathbb{Z}^{2}$ for which there are no $\mathscr{A}$-hypergeometric Laurent polynomials of exponent $\alpha$, is the EulerJacobi cone,

$$
\mathscr{E} \mathscr{F}:=\left\{\left(\alpha_{1}, \alpha_{2}\right) \in \mathbb{Z}^{2}: d \alpha_{1}<\alpha_{2}<0\right\} .
$$

(ii) For arbitrary $\mathscr{A}$ as in (0.4), we have $I(\mathscr{A}) \subset \mathscr{C}, E(\mathscr{A}) \subset \mathscr{C}$, and $\mathscr{E} \mathscr{E} \subset J(\mathscr{A})$.

(iii) The conditions (1.10) and (1.11) are far from being sharp. It is easy to see, for example, that for $\alpha \in \mathscr{C}, \Psi_{0}^{\mathscr{A}}(\alpha ; x) \neq 0$ and $\Psi_{d}^{\mathscr{A}}(\alpha ; x) \neq 0$ imply that $k_{1} \alpha_{1}<\alpha_{2}<$ $k_{m} \alpha_{1}$. In particular, if $m=1, k_{1}=k_{m}$ and there is no $\alpha \in \mathscr{C}$ for which both $\Psi_{0}^{A}(\alpha ; x)$ and $\Psi_{d}^{A}(\alpha ; x)$ are nontrivial. Hence, there is for each $\alpha$ at most one, up to constant multiple, Laurent solution of exponent $\alpha$. Note also that the toric ring $R$ is always Cohen-Macaulay but it is normal if and only if $d=2$.

(iv) It is not hard to prove (see, for example, [2, Lemma 3.12]) that there exists $v \in \mathscr{A} \cdot \mathbb{N}^{m+2}$ such that $v+\mathscr{C} \subset I(\mathscr{A})$. Thus, for $\alpha \in v+\mathscr{C}$, there is a unique $\mathscr{A}$ hypergeometric Laurent polynomial, and it is given by (1.1). Moreover, when this observation is combined with the inequalities in (iii), it follows that the set $E(\mathscr{A})$ is finite.

1.8. Examples. We exhibit two examples where $E(\mathscr{A}) \neq \emptyset$ and, consequently, the associated toric ring $R$ is not Cohen-Macaulay.

(i) This is the "running example" in [23]. Let

$$
\mathscr{A}=\left(\begin{array}{llll}
1 & 1 & 1 & 1 \\
0 & 1 & 3 & 4
\end{array}\right)
$$

The exponent $\alpha=(1,2)$ is the unique element in $\mathscr{C}$ such that $\alpha \notin I(\mathscr{A})$ and $\alpha_{1}<$ $\alpha_{2}<3 \alpha_{1}$. Both $\Psi_{0}^{A A}((1,2) ; x)=(-1 / 2)\left(x_{1}^{2} / x_{0}\right)$ and $\Psi_{4}^{A A}((1,2) ; x)=(-1 / 2)\left(x_{3}^{2} / x_{4}\right)$ are nontrivial.

The element $\beta=(2,3) \in F_{0}(\mathscr{A}) \cap F_{4}(\mathscr{A})$. Therefore both $\Psi_{0}^{\mathscr{A}}(\beta ; x)=(-1 / 6) x_{1}^{3} / x_{0}$ and $\Psi_{4}^{A}(\beta ; x)=(-1 / 2) x_{1} x_{3}^{2} / x_{4}$ are nontrivial. However, since $\beta=\mathscr{A} \cdot\left(e_{0}+e_{3}\right) \in$ $I(\mathscr{A})$, it follows from Lemma 1.3 that neither $\Psi_{0}^{\mathscr{A}}(\beta ; x)$ nor $\Psi_{d}^{\mathscr{A}}(\beta ; x)$ is $\mathscr{A}$-hypergeometric, and the only $\mathscr{A}$-hypergeometric Laurent polynomials of exponent $\beta$ are the multiples of the polynomial $\Phi^{\mathscr{A}}(\beta ; x)=x_{0} x_{3}$.

(ii) Consider the system associated with the matrix

$$
\mathscr{A}=\left(\begin{array}{ccccc}
1 & 1 & 1 & 1 & 1 \\
0 & 6 & 7 & 13 & 14
\end{array}\right)
$$

and $\alpha=(2,18) \notin I(\mathscr{A})$. We have

$$
\Psi_{0}^{A}((2,18) ; x)=-\frac{1}{6} \frac{x_{6}^{3}}{x_{0}} ; \quad \Psi_{14}^{A}((2,18) ; x)=-\frac{1}{2} \frac{x_{6} x_{13}^{2}}{x_{14}}+\frac{1}{6} \frac{x_{7} x_{13}^{3}}{x_{14}^{2}} .
$$


It follows from (1.10) and (1.11) that there are no $\mathscr{A}$-hypergeometric Laurent polynomials whose exponent $\alpha$ is in the Euler-Jacobi cone (1.13). In fact, as the following result shows, there are no rational solutions with exponent in that region.

THeOREM 1.9. The A-hypergeometric system associated with the matrix (0.4) has no rational solutions whose exponent $\alpha$ lies in the Euler-Jacobi cone.

Given $\alpha \in \mathbb{Z}^{2}$, we denote by $\mathscr{R}(\alpha)$ the vector space of rational $\mathscr{A}$-hypergeo- metric functions of exponent $\alpha$. Before proving Theorem 1.9, we note the following consequence.

THEOREM 1.10. The only rational solutions of the A-hypergeometric system associated with the matrix (0.4) are the Laurent polynomial solutions described by Proposition 1.1 and Theorem 1.4.

Proof. Suppose $\varphi \in \mathscr{R}(\alpha)$. For $\ell \in \mathbb{N}$ sufficiently large, $\beta=\alpha-\ell\left(1, k_{1}\right)$ lies in the Euler-Jacobi cone. Then $D_{k_{1}}^{\ell} \varphi \in \mathscr{R}(\beta)$, and therefore it is identically zero by Theorem 1.9. Hence, $\varphi$ is polynomial in $x_{k_{1}}$, and by Proposition 1.2 it must be a Laurent polynomial.

1.11. Example. Theorem 1.10 is not true for $n>1$. It fails already in the simplest 2-dimensional situation: Consider the hypergeometric system associated with the matrix

$$
\mathscr{A}=\left(\begin{array}{llll}
1 & 1 & 1 & 1 \\
0 & 1 & 0 & 1 \\
0 & 0 & 1 & 1
\end{array}\right)
$$

The lattice $\mathscr{L}$ has rank 1 ; in fact $\mathscr{L}=\mathbb{Z} \cdot(1,-1,-1,1)^{T}$, and the system $(0.1)-(0.2)$ is equivalent to Gauss's classical hypergeometric equation. The function $1 /\left(x_{1} x_{4}-\right.$ $\left.x_{2} x_{3}\right)$ is a solution with parameters $(-2,-1,-1)$.

Proof of Theorem 1.9. The proof is by induction on $m$. We begin by considering the case $m=1$ and write, for simplicity, $k_{1}=k$. Note that in this case the lattice $\mathscr{L}$ has rank 1 and is generated by $\omega:=(d-k) e_{0}-d e_{k}+k e_{d}$. In particular, for appropriate values of $\alpha$, we can write the $\mathscr{A}$-hypergeometric functions in terms of classical hypergeometric functions (see [9, §3.1]).

The discriminant of the generic polynomial $x_{0}+x_{k} t^{k}+x_{d} t^{d}$ is, up to factors that are powers of $x_{0}$ and $x_{d}$,

$$
\Delta(x)=d^{d} x_{0}^{d-k} x_{d}^{k}+(-1)^{d-1} k^{k}(d-k)^{d-k} x_{k}^{d}=c x_{k}^{d}(1-\lambda z),
$$

where $c=(-1)^{d-1} k^{k}(d-k)^{d-k}, z=x^{\omega}=x_{0}^{d-k} x_{k}^{-d} x_{d}^{k}$, and

$$
\lambda=\frac{(-1)^{d} d^{d}}{k^{k}(d-k)^{d-k}} .
$$

Suppose now that $R(x)=P(x) / Q(x) \in \mathscr{R}(\alpha)$ with $\alpha \in \mathscr{E} \mathscr{g}$, that is, $d \alpha_{1}<\alpha_{2}<0$. 
Note that both $P$ and $Q$ are bihomogeneous relative to the $\mathbb{Z}^{2}$-degree defined by $\mathscr{A}$. We can then write $P(x)=x^{v} P_{1}(z)$, where $P_{1}$ is a polynomial and $P_{1}(0) \neq 0$. Thus, up to a constant,

$$
R(x)=x^{u} \frac{P_{1}(z)}{(1-\lambda z)^{r}}=x^{u} \sum_{j \geq 0} c_{j} z^{j}=x^{u} \sum_{j \geq 0} c_{j} x^{j \omega},
$$

with $c_{0} \neq 0$ and $\mathscr{A} \cdot u=\alpha$.

Since $R$ is in the kernel of the differential operator

$$
\mathscr{D}_{\omega}=D_{0}^{d-k} D_{d}^{k}-D_{k}^{d}
$$

the coefficients in (1.14) satisfy the relation

$c_{j} \prod_{\ell=0}^{d-1}\left(u_{k}-j d-\ell\right)=c_{j+1} \prod_{\ell^{\prime}=0}^{d-k-1}\left(u_{0}+(j+1)(d-k)-\ell^{\prime}\right) \prod_{\ell^{\prime \prime}=0}^{k-1}\left(u_{d}+(j+1) k-\ell^{\prime \prime}\right)$,

for all $j \in \mathbb{Z}$. Setting $j=-1$ we get

$$
0=c_{0} \prod_{\ell^{\prime}=0}^{d-k-1}\left(u_{0}-\ell^{\prime}\right) \prod_{\ell^{\prime \prime}=0}^{k-1}\left(u_{d}-\ell^{\prime \prime}\right)
$$

which implies that either $0 \leq u_{0} \leq d-k-1$ or $0 \leq u_{d} \leq k-1$. On the other hand, $k u_{k}+d u_{d}=\alpha_{2}<0$ and $d u_{0}+(d-k) u_{k}=d \alpha_{1}-\alpha_{2}<0$, which means that in either case $u_{k}<0$. Therefore, the left-hand side of (1.16) is never zero and, consequently, neither is the right-hand side. This implies that $u_{0} \geq 0$ and $u_{d} \geq 0$ and for all $j \geq 0$,

$$
c_{j}=c(-1)^{j d} \frac{\left(-u_{k}+j d\right) !}{\left(u_{0}+j(d-k)\right) !\left(u_{d}+j k\right) !},
$$

for some constant $c$.

But a function with such an expansion may not be rational. Indeed, Stirling's formula implies that, asymptotically, $c_{j} \sim \mu j^{-\alpha_{1}} \lambda^{j} / \sqrt{j}$, for some constant $\mu$. On the other hand, for a rational function $R$ whose denominator is a power of $(1-\lambda z)$, we would have $c_{j}=p(j) \lambda^{j}$, with $p$ a polynomial. This completes the proof of Theorem 1.9 in the case $m=1$.

Assume now that Theorem 1.9 and its consequence Theorem 1.10 are valid for $m-1, m \geq 2$, and consider the $\mathscr{A}$-hypergeometric system associated with the matrix $\mathscr{A}$ in $(0.4)$.

Let $R(x)$ be a rational solution with parameter $\alpha$ in the Euler-Jacobi cone; that is, $\alpha=\left(\alpha_{1}, \alpha_{2}\right)$, with $d \alpha_{1}<\alpha_{2}<0$. Suppose we can write $R(x)=P(x) /\left(x_{d}^{r} Q(x)\right) ; r>$ 0 , and assume that $x_{d}$ does not divide $P(x)$ or $Q(x)$. Again, let $x^{\prime}=\left(x_{0}, x_{k_{1}}, \ldots, x_{k_{m}}\right)$, 
and write $Q(x)=\sum_{k=0}^{r} q_{k}\left(x^{\prime}\right) x_{d}^{k}$, with $q_{0}\left(x^{\prime}\right) \neq 0$, and set $c_{k}\left(x^{\prime}\right)=-q_{k}\left(x^{\prime}\right) / q_{0}\left(x^{\prime}\right)$. Then,

$$
R(x)=\frac{P(x)}{x_{d}^{r} \cdot q_{0}\left(x^{\prime}\right)}\left(1+\sum_{m=1}^{\infty}\left(\sum_{k=1}^{r} c_{k}\left(x^{\prime}\right) x_{d}^{k}\right)^{m}\right)=\sum_{\ell \geq-r} A_{\ell}\left(x^{\prime}\right) x_{d}^{\ell} .
$$

By inductive assumption, since $A_{\ell}\left(x^{\prime}\right)$ is a rational $\mathscr{B}$-hypergeometric function, it must be a Laurent polynomial as described by Theorem 1.4. (Note that even though we may have $\ell=\operatorname{gcd}\left(k_{1}, \ldots, k_{m}\right)>1$, we may easily reduce to the system associated with a matrix where $k_{i}$ has been replaced by $k_{i} / \ell$.) Set $x_{d}=0$ and consider the nontrivial rational function $A_{-r}\left(x^{\prime}\right)=P\left(x^{\prime}, 0\right) / Q\left(x^{\prime}, 0\right)$ that has $\mathscr{B}$-exponent $\alpha^{\prime}(r)=$ $\left(\alpha_{1}+r, \alpha_{2}+d r\right)$. It is clear that $\alpha^{\prime}(r) \notin E(\mathscr{B})$, and hence there may be, up to constant, at most one Laurent solution with exponent $\alpha^{\prime}(r)$. We distinguish the two possible cases.

(i) Suppose $A_{-r}\left(x^{\prime}\right)$ is a nonzero multiple of $\Psi_{k_{m}}^{\mathscr{R}}\left(\alpha^{\prime}(r), x^{\prime}\right)$. Then it contains a nonzero term of the form

$$
\frac{\hat{x}^{u}}{x_{k_{m}}^{s}}, \quad s>0,
$$

where $\hat{x}^{u}$ is a monomial with positive exponents involving only the variables $x_{0}$, $x_{k_{1}}, \ldots, x_{k_{m-1}}$. Thus, a Laurent series expansion of $R(x)$, as a function of $x_{d}$, has a nonzero term of the form

$$
\frac{\hat{x}^{u}}{x_{k_{m}}^{s} x_{d}^{r}}, \quad r>0, s>0 .
$$

Successive applications of the fact that $R$ is in the kernel of the operator (1.15), with $k_{m}$ in the place of $k$, yields that $R$ must also contain a nonzero term whose derivative $D_{0}^{j\left(d-k_{m}\right)} D_{d}^{j k_{m}}$ is a multiple of

$$
\frac{\hat{x}^{u}}{x_{k_{m}}^{s+d j} x_{d}^{r}}, \quad r>0, s>0
$$

for all $j \geq 0$, which is impossible as soon as $j k_{m} \geq r$.

(ii) Suppose $A_{-r}\left(x^{\prime}\right)$ is a nonzero multiple of $\Psi_{0}^{\Re_{B}}\left(\alpha^{\prime}(r), x^{\prime}\right)$. Then it contains a nonzero term of the form

$$
\frac{\check{x}^{u}}{x_{0}^{s}}, \quad s>0,
$$

where $\check{x}^{u}$ is a monomial with positive exponents involving only the variables $x_{k_{1}}, \ldots, x_{k_{m}}$, which implies that a Laurent series expansion of $R(x)$, as a function of $x_{d}$, has a nonzero term of the form

$$
\frac{\check{x}^{u}}{x_{0}^{s} x_{d}^{r}}, \quad r>0, s>0 .
$$

But, since the operator (1.15), with $k_{m}$ in the place of $k$, must vanish on $R$, there must 
also be a nonzero term of the form

$$
\frac{\check{x}^{u} x_{k_{m}}^{d}}{x_{0}^{s+d-k_{m}} x_{d}^{r+k_{m}}}
$$

which contradicts the index bound in (1.17).

By symmetry, we can then assume that $R(x)=P(x) / Q(x)$ and neither $x_{d}$ nor $x_{0}$ divide $Q$. Thus, $R(x)$ is written as in (1.17) with $r=0$. For each $\ell \geq 0, A_{\ell}\left(x^{\prime}\right)$ is a solution of the $\mathscr{B}$-hypergeometric system with exponent $\left(\alpha_{1}-\ell, \alpha_{2}-d \ell\right)$. By inductive hypothesis, these must be Laurent polynomials, and it is easy to check that the only possible denominators are powers of $x_{k_{m}}$. Thus, only powers of $x_{k_{m}}$ may appear in the denominator of the above expansion for $R$, which implies that $q_{0}\left(x^{\prime}\right)$ must be of the form $x_{k_{m}}^{d}$, and, therefore, $Q(x)$ has bidegree $\left(d, k_{m} d\right)$. On the other hand, a symmetric argument would imply that $Q(x)$ must contain a term of the form $x_{k_{1}}^{e}$, and, hence, $Q(x)$ should have bidegree $\left(e, k_{1} e\right)$. Since $m>1$, this implies that $Q(x)$ has degree zero, but then $R(x)$ is a polynomial solution, which is impossible since, being in the Euler-Jacobi cone, $\alpha \notin \mathscr{A} \cdot \mathbb{N}^{m+2}$.

As we noted before, given $s \in \mathbb{Z}$, the sum (0.6), $p_{s}(x)=\rho_{1}^{s}(x)+\cdots+\rho_{d}^{s}(x)$, of the powers of the roots of the generic polynomial (0.5) is a rational $\mathscr{A}$-hypergeometric function with exponent $(0,-s)$. By Theorem 1.10 it must be a Laurent polynomial and, therefore, expressible in terms of $\Psi_{0}^{\mathscr{A}}$ and $\Psi_{d}^{\mathscr{A}}$. In fact, we have the following result.

Corollary 1.12. For $s>0$,

$$
p_{s}(x)=s \cdot \Psi_{d}^{A}((0,-s) ; x)=s \cdot \sum_{r=1}^{s}(-1)^{r}(r-1) ! \frac{\Phi^{\mathscr{B}}\left((r, r d-s) ; x^{\prime}\right)}{x_{d}^{r}},
$$

while for $s<0$,

$$
p_{s}(x)=s \cdot \Psi_{0}^{A}((0,-s) ; x)=s \cdot \sum_{r=1}^{s}(-1)^{r}(r-1) ! \frac{\Phi^{\mathscr{C}}((r,-s) ; \tilde{x})}{x_{0}^{r}} .
$$

Proof. It suffices to consider the normal case, $d=m+1$, and then set the appropriate variables equal to zero. For $s>0$, it follows from (1.11) that $p_{s}(x)$ must be a multiple of $\Psi_{d}^{A d}((0,-s) ; x)$. It is easy to see that the value of the multiple must be $s$ by specialization to the case when $f(x ; t)=t^{d}+t^{d-1}$. The statement for $s<0$ follows by symmetry after observing that with the change of variables (1.6), the polynomial $\hat{f}(y ; \tau)$ associated with the matrix $\hat{A}$ in $(1.5)$ is given by $\hat{f}(y ; \tau)=\tau^{d} \cdot f\left(x ; \tau^{-1}\right)$ and, consequently, its roots are the inverse of those of $f$.

1.13. Remarks. (i) Note that each term in the right-hand side of (1.18) is of total degree zero and, therefore, we may express $p_{s}(x)$ as a polynomial in $x_{d-j} / x_{d}=$ 
$(-1)^{j} \sigma_{j}\left(\rho_{1}, \ldots, \rho_{d}\right), j=1, \ldots, d$, where $\sigma_{j}$ is the $j$ th elementary symmetric polynomial. This yields the classical Girard formulas.

(ii) As we noted in the introduction, the total sum of the local residues (0.7) gives a rational $\mathscr{A}$-hypergeometric function with exponent $(-a,-b)$ and hence, as in Corollary 1.12, it must be a multiple of $\Psi_{d}^{A A}((-a,-b) ; x)$, if $b>0$, and of $\Psi_{0}^{A}((-a,-b) ; x)$, if $b \leq 0$. In particular, for $a=1, b>0$, we have

$$
\sum_{\rho} \operatorname{Res}_{\rho(x)}\left(\frac{t^{b}}{f(x ; t)} \frac{d t}{t}\right)= \begin{cases}0, & \text { if } 0<b<d \\ -\Psi_{d}^{A}((-1,-b) ; x), & \text { if } b \geq d .\end{cases}
$$

We end this section with a result that should be seen as a complement to (1.10) and (1.11) and which is of use in $\S 2$.

Proposition 1.14. Let $\alpha=\left(\alpha_{1}, \alpha_{2}\right) \in \mathbb{Z}^{2} \backslash I(\mathscr{A})$, and set $s=s(\alpha):=d \alpha_{1}-\alpha_{2}$.

(i) If $s>0$, then $\Psi_{d}^{A}(\alpha ; x)=0$ if and only if $p_{s}(x)=0$.

(ii) If $\alpha_{2}>0$, then $\Psi_{0}^{A}(\alpha ; x)=0$ if and only if $p_{-\alpha_{2}}(x)=0$.

Proof. It suffices to prove the first statement and then deduce (ii) by symmetry. Suppose $\beta \in \mathbb{Z}^{2}$ with $\beta_{1}=\alpha_{1}-r, r>0$, and $s(\beta)=s(\alpha)$. Then, if $\Psi_{d}^{A}(\alpha ; x)=0$, we have by $(1.12) \Psi_{d}^{A}(\beta ; x)=D_{d}^{r} \Psi_{d}^{A}(\alpha ; x)=0$. But, if $\Psi_{d}^{A}(\alpha ; x) \neq 0$, then the same argument implies that $\Psi_{d}^{A}(\beta ; x) \neq 0$ since the dependence of $\Psi_{d}^{A}(\alpha ; x)$ on $x_{d}$ is not polynomial. Hence, given $\alpha, \beta \in \mathbb{Z}^{2} \backslash \mathscr{A} \cdot \mathbb{N}^{m+2}$ such that $s(\alpha)=s(\beta)$, $\Psi_{d}^{A A}(\alpha ; x)=0$ if and only if $\Psi_{d}^{A d}(\beta ; x)=0$. The result now follows from the fact that $\Psi_{d}^{A}((0,-s) ; x)=s p_{s}(x)$.

2. Algebraic solutions. In this section we introduce a family, $\psi_{\rho}$, of local algebraic solutions of the $\mathscr{A}$-hypergeometric system associated with a monomial curve. These solutions, which are given in terms of the roots $\rho(x)$ of the generic polynomial (0.5), play a central role in $\S 3$ when we compute the holonomic rank and construct a basis of local solutions for all exponents $\alpha \in \mathbb{Z}^{2}$.

Let $\mathscr{A}$ be as in (0.4). Given an open set $\mathcal{U} \subseteq \mathbb{C}^{m+2}$, let $\mathscr{H}(\alpha)(\mathcal{U})$ denote the space of solutions, holomorphic on $\mathcal{U}$, of the $\mathscr{A}$-hypergeometric system with exponent $\alpha$, and let $\mathscr{H}_{\text {alg }}(\alpha)(U)$ denote the subspace of algebraic solutions. We drop the reference to the open set $U$ whenever we are only interested in the space of local solutions around a generic point. Let $\rho(x)$ be a root of the polynomial $f(x ; t)$ defined by $(0.5)$, holomorphic for $x$ in some simply connected open subset $u \subset \mathbb{C}^{m+2} \backslash \Sigma$.

Given $\alpha=\left(\alpha_{1}, \alpha_{2}\right) \in \mathbb{Z}^{2}, \alpha_{1} \geq 0$, we define

$$
\psi_{\rho}(\alpha ; x):=\sum_{\substack{i=0 \\ i \neq \alpha_{2}}}^{d \alpha_{1}} \Phi^{\mathscr{A}}\left(\left(\alpha_{1}, i\right) ; x\right) \frac{\rho^{i-\alpha_{2}}(x)}{i-\alpha_{2}}+\Phi^{\mathscr{A}}(\alpha ; x) \log (\rho(x)) .
$$

Note that the condition $i \neq \alpha_{2}$ is automatically satisfied when $\alpha_{2}<0$ or $\alpha_{2}>d \alpha_{1}$, 
and that for $\alpha_{1}=0, \alpha_{2} \neq 0, \psi_{\rho}(\alpha)$ is just $\rho^{-\alpha_{2}} /\left(-\alpha_{2}\right)$. (If there is no ambiguity, we drop any reference to the variable $x$.)

For $\alpha \notin I(\mathscr{A})$, the hypergeometric polynomial $\Phi^{\mathscr{A}}(\alpha ; x)$ vanishes, and $\psi_{\rho}(\alpha)$ is an algebraic function. This is the case that is of interest in this section; however, in $\S 3$ we need (2.1) for arbitrary $\alpha \in \mathbb{Z}^{2}$, and it is, therefore, economical to work in this slightly more general setting. We extend the definition of $\psi_{\rho}(\alpha)$ to the case $\alpha_{1}<0$ in (2.6).

Proposition 2.1. Let $\alpha, \alpha^{\prime} \in \mathbb{Z}^{2}$ and assume that $\alpha_{1}, \alpha_{1}^{\prime} \geq 0$. Let $u, u^{\prime} \in \mathbb{N}^{m+2}$ be such that $\alpha-\mathscr{A} \cdot u=\alpha^{\prime}-\mathscr{A} \cdot u^{\prime}$. Then

$$
D_{u} \psi_{\rho}(\alpha)=D_{u^{\prime}} \psi_{\rho}\left(\alpha^{\prime}\right)
$$

Proof. In order to prove (2.2), we show, as a first step, that if $\alpha=\left(\alpha_{1}, \alpha_{2}\right)$ and $\alpha_{1}>0$, then

$$
\frac{\partial}{\partial x_{\ell}}\left(\psi_{\rho}(\alpha)\right)=\psi_{\rho}\left(\alpha-\mathscr{A} \cdot e_{\ell}\right)
$$

Since, by (1.2), $\partial\left(\Phi^{\mathscr{A}}(\alpha)\right) / \partial x_{\ell}=\Phi^{\mathscr{A}}\left(\alpha-\mathscr{A} \cdot e_{\ell}\right)$, we have

$$
\begin{aligned}
\frac{\partial}{\partial x_{\ell}}\left(\psi_{\rho}(\alpha)\right)= & \sum_{\substack{i=0 \\
i \neq \alpha_{2}}}^{d \alpha_{1}} \Phi^{\mathscr{A}}\left(\left(\alpha_{1}-1, i-\ell\right)\right) \frac{\rho^{i-\alpha_{2}}}{i-\alpha_{2}}+\Phi^{\mathscr{A}}\left(\alpha-\mathscr{A} \cdot e_{\ell}\right) \log (\rho) \\
& +\sum_{\substack{i=0 \\
i \neq \alpha_{2}}}^{d \alpha_{1}} \Phi^{\mathscr{A}}\left(\left(\alpha_{1}, i\right)\right) \rho^{i-\alpha_{2}-1} \frac{\partial \rho}{\partial x_{\ell}}+\Phi^{\mathscr{A}}(\alpha) \rho^{-1} \frac{\partial \rho}{\partial x_{\ell}} .
\end{aligned}
$$

Note that the last two terms in the expression above cancel, since $f^{\alpha_{1}}(x ; \rho(x))=0$ implies that

$$
\sum_{\substack{i=0 \\ i \neq \alpha_{2}}}^{d \alpha_{1}} \Phi^{\mathscr{A}}\left(\alpha_{1}, i\right) \rho^{i}=-\Phi^{\mathscr{A}}(\alpha) \rho^{\alpha_{2}}
$$

On the other hand, setting $j=i-\ell$, the right-hand side of (2.4) becomes

$$
\sum_{\substack{j=0 \\ j \neq \alpha_{2}-\ell}}^{d\left(\alpha_{1}-1\right)} \Phi^{\mathscr{A}}\left(\left(\alpha_{1}-1, j\right)\right) \frac{\rho^{j-\left(\alpha_{2}-\ell\right)}}{j-\left(\alpha_{2}-\ell\right)}+\Phi^{\mathscr{A}}\left(\alpha-\mathscr{A} \cdot e_{\ell}\right) \log (\rho)=\psi_{\rho}\left(\alpha-\mathscr{A} \cdot e_{\ell}\right)
$$

where we have used that the hypergeometric polynomial $\Phi^{\mathscr{A}}\left(\left(\alpha_{1}-1, j\right)\right)$ vanishes for $j<0$ or $j>d\left(\alpha_{1}-1\right)$. This proves (2.3).

Applying (2.3) successively we may assume that $\alpha_{1}=\alpha_{1}^{\prime}=0$. Suppose $\alpha=$ $(0,-s), s \neq 0$, so that $\Phi^{\mathscr{A}}(\alpha)=0$ and $\psi_{\rho}(\alpha)=\rho^{s} / s$. Given $u \in \mathbb{N}^{m+2}$ we want to 
compute $D_{u}\left(\psi_{\rho}(\alpha)\right)$. Locally on $x$ we can write

$$
\begin{aligned}
D_{u}\left(\frac{2 \pi i}{s} \rho^{s}\right) & =\int_{\Gamma} \frac{t^{s}}{s} D_{u}\left(\frac{f^{\prime}(x ; t)}{f(x ; t)}\right) d t \\
& =\int_{\Gamma} \frac{d}{d t}\left(\frac{t^{s}}{s} D_{u}(\log f(x ; t))\right) d t-\int_{\Gamma} t^{s-1} D_{u}(\log f(x ; t)) d t \\
& =(-1)^{\beta_{1}-1}\left(\beta_{1}-1\right) ! \int_{\Gamma} t^{s-1} \frac{t^{\beta_{2}}}{f^{\beta_{1}}} d t
\end{aligned}
$$

where $\mathscr{A} \cdot u=\left(\beta_{1}, \beta_{2}\right)$ and $\Gamma$ is a sufficiently small loop in the complex plane. Thus $D_{u}\left(\psi_{\rho}(\alpha)\right)$ depends only on the pair $\left(\beta_{1}, \beta_{2}+s\right)=-(\alpha-\mathscr{A} \cdot u)$. If $\alpha=(0,0)$, $\psi_{\rho}((0,0))=\log (\rho)$ and we can show that $D_{u}(\log (\rho))$ depends only on $\mathscr{A} \cdot u$, arguing as above with $\log (t)$ taking the place of $t^{s} / s$. This completes the proof.

Proposition 2.1 now allows us to extend the definition of $\psi_{\rho}(\alpha)$ to the case $\alpha_{1}<0$. Indeed, let $u \in \mathbb{N}^{m+2}$ be such that $\mathscr{A} \cdot u=\left(\beta_{1}, \beta_{2}\right)$, with $\beta_{1} \geq-\alpha_{1}$, and set

$$
\psi_{\rho}(\alpha):=D_{u}\left(\psi_{\rho}(\alpha+\mathscr{A} \cdot u)\right)
$$

Clearly, this definition does not depend on the choice of $u$, and for any $\alpha \in \mathbb{Z}^{2}$, $u \in \mathbb{N}^{m+2}$,

$$
D_{u}\left(\psi_{\rho}(\alpha)\right)=\psi_{\rho}(\alpha-\mathscr{A} \cdot u) .
$$

If $\alpha \notin I(\mathscr{A})$ we can choose $u$ so that $\alpha+\mathscr{A} \cdot u \notin I(\mathscr{A})$ either, and, hence, $\psi_{\rho}(\alpha)$ is an algebraic function.

TheOREM 2.2. For $\alpha \in \mathbb{Z}^{2} \backslash I(\mathscr{A})$ the algebraic functions $\psi_{\rho}(\alpha)$ are $\mathscr{A}$-hypergeometric.

Proof. Given (2.6), it suffices to consider the case $\alpha_{1} \geq 0$. The hypergeometric polynomials $\Phi^{\mathscr{A}}\left(\left(\alpha_{1}, i\right) ; x\right)$ are $\mathscr{A}$-bihomogeneous of bidegree $\left(\alpha_{1}, i\right)$ while the powers $\rho^{i-\alpha_{2}}(x)$ have bidegree $\left(0, \alpha_{2}-i\right)$. Hence, $\psi_{\rho}(\alpha)$ satisfies the homogeneity equations (0.2) with exponent $\alpha$. On the other hand, it is an immediate consequence of Proposition 2.1 that $\psi_{\rho}(\alpha)$ satisfies the higher-order equations $(0.1)$.

Let now $U \subset \mathbb{C}^{m+2} \backslash \Sigma$ be a simply connected open set, and let $\rho_{1}(x), \ldots, \rho_{d}(x)$ be distinct roots of the polynomial $f(x ; t)$, holomorphic for $x \in \mathcal{U}$. Let $\alpha \in \mathbb{Z}^{2} \backslash I(\mathscr{A})$, and set $\psi_{j}(\alpha):=\psi_{\rho_{j}}(\alpha) \in \mathscr{H}_{\mathrm{alg}}(\alpha)(\mathcal{U})$. The function

$$
\Psi^{\mathscr{A}}(\alpha):=\psi_{1}(\alpha)+\cdots+\psi_{d}(\alpha)
$$

is then a rational solution of the $\mathscr{A}$-hypergeometric system with parameters $\alpha$. It follows from (2.7) that, for any $u \in \mathbb{N}^{m+2}$,

$$
D_{u}\left(\Psi^{\mathscr{A}}(\alpha)\right)=\Psi^{\mathscr{A}}(\alpha-\mathscr{A} \cdot u) .
$$


Proposition 2.3. Let $\alpha \in \mathbb{Z}^{2} \backslash I(\mathscr{A})$. Then,

(i) $\Psi^{A}(\alpha ; x)=\Psi_{d}^{A}(\alpha ; x)+\Psi_{0}^{A}(\alpha ; x)$, defined as in (1.7) and (1.8);

(ii) $\Psi^{A}(\alpha)=0$ if and only if there exist no nontrivial rational $A$-hypergeometric functions with parameter $\alpha$;

(iii) if $\alpha=\left(\alpha_{1}, \alpha_{2}\right)$ and $\alpha_{1}>0$, then

$$
\Psi_{0}^{A}(\alpha ; x)=\sum_{\substack{i=0 \\ i<\alpha_{2}}}^{d \alpha_{1}} \Phi^{\mathscr{A}}\left(\left(\alpha_{1}, i\right) ; x\right) \Psi_{0}^{\mathscr{A}}\left(\left(0, \alpha_{2}-i\right) ; x\right),
$$

and

$$
\Psi_{d}^{\mathscr{A}}(\alpha ; x)=\sum_{\substack{i=0 \\ i>\alpha_{2}}}^{d \alpha_{1}} \Phi^{\mathscr{A}}\left(\left(\alpha_{1}, i\right) ; x\right) \Psi_{d}^{\mathscr{A}}\left(\left(0, \alpha_{2}-i\right) ; x\right) .
$$

Proof. By Theorem 1.4(iii), $\Psi^{\mathscr{A}}(\alpha)$ is a linear combination $\lambda \Psi_{d}^{A}(\alpha ; x)+$ $\mu \Psi_{0}^{A A}(\alpha ; x)$. Moreover, if $\alpha_{1}=0$, the result follows from (1.18) and (1.19). Therefore, computing derivatives with respect to $x_{d}$, the result follows for $s(\alpha)=d \alpha_{1}-\alpha_{2}>0$, $\alpha_{1}<0$, where $\Psi_{0}^{A}(\alpha ; x)=0$. By symmetry, (i) also holds for $\alpha_{2}>0, \alpha_{1}<0$.

If $\alpha \in \mathbb{Z}^{2} \backslash I(\mathscr{A})$ is such that $\Psi_{d}^{A}(\alpha ; x) \neq 0$, then

$$
\Psi^{\mathscr{A}}((0,-s(\alpha)))=\frac{\partial^{\alpha_{1}} \Psi^{\mathscr{A}}(\alpha)}{\partial x_{d}^{\alpha_{1}}}=\lambda \frac{\partial^{\alpha_{1}} \Psi_{d}^{\mathscr{A}}(\alpha)}{\partial x_{d}^{\alpha_{1}}}=\lambda \Psi_{d}^{\mathscr{A}}((0,-s(\alpha))),
$$

which implies $\lambda=1$ since, because of Proposition $1.14, \Psi_{d}^{A}((0,-s(\alpha))) \neq 0$. A similar argument shows that if $\Psi_{0}^{A}(\alpha ; x) \neq 0$, then $\mu=1$.

The second assertion is an immediate consequence of Theorem 1.4, while the identities (2.9) and (2.10) follow from (i), together with (1.18) and (1.19).

We now determine the dimension of the subspace of algebraic hypergeometric functions over $U$ spanned by $\psi_{1}(\alpha), \ldots, \psi_{d}(\alpha), \alpha \notin I(\mathscr{A})$.

Theorem 2.4. For $\alpha \in \mathbb{Z}^{2} \backslash I(\mathscr{A})$, the A-hypergeometric functions $\psi_{1}(\alpha), \ldots$, $\psi_{d}(\alpha)$ span a linear space of dimension at least $d-1$. Moreover, they are linearly dependent if and only if $\Psi^{\mathfrak{A}}(\alpha)=0$.

Before giving the proof of Theorem 2.4, we first recall the construction by Gel'fand, Zelevinsky, and Kapranov [9] of $\Gamma$-series solutions for the $\mathscr{A}$-hypergeometric system and the expressions obtained by Sturmfels [22] for the roots of $f(x ; t)$ in terms of them. We begin by considering the normal case, and eventually we specialize coefficients to study the general case. We only need to consider the coarsest triangulation of the polytope $P$, the convex hull of $\mathscr{A}$ and the origin, that is, the one consisting of the single simplex $P$. As before, we let $\mathscr{L}$ stand for the integral kernel of $\mathscr{A}$, that is, the sublattice of elements $v \in \mathbb{Z}^{d+1}$ such that $\mathscr{A} \cdot v=0$. Given $u \in \mathbb{Q}^{d+1}$, we define the formal power series 


$$
\left[x_{0}^{u_{0}} x_{1}^{u_{1}} \cdots x_{d}^{u_{d}}\right]:=\sum_{v \in \mathscr{L}} \prod_{i=0}^{d}\left(\gamma\left(u_{i}, v_{i}\right) x_{i}^{u_{i}+v_{i}}\right)
$$

where, for any rational number $u$ and any integer $v$, we write

$$
\gamma(u, v):= \begin{cases}1, & \text { if } v=0, \\ u(u-1)(u-2) \cdots(u+v+1), & \text { if } v<0, \\ 0, & \text { if } u \text { is a negative integer and } u \geq-v, \\ \frac{1}{(u+1)(u+2) \cdots(u+v)}, & \text { otherwise. }\end{cases}
$$

If $u$ has no negative integer coordinates or is of the form $(0, \ldots, 0,1,-1,0, \ldots, 0)$, the series $\left[x_{0}^{u_{0}} \cdots x_{d}^{u_{d}}\right]$ is a formal solution of the $\mathscr{A}$-hypergeometric system with parameters $\mathscr{A} \cdot u \in \mathbb{Q}^{2}$ (see [9, Lemma 1] and [22, Lemma 3.1]). Moreover, if at most two of the exponents $u_{i}$ are nonintegers, the series (2.11) converges in a suitable open subset of $\mathbb{C}^{d+1} \backslash \Sigma$ (see [9]).

Let $\xi_{1}, \ldots, \xi_{d}$ be the $d$ th roots of -1 and set $\rho_{i}(x):=\sum_{a=1}^{d} \xi_{i}^{a} \cdot \sigma_{a}(x)$, where

$$
\sigma_{1}(x):=\left[x_{0}^{1 / d} x_{d}^{-(1 / d)}\right] ; \quad \sigma_{a}(x):=\frac{1}{d} \cdot\left[x_{a-1} x_{0}^{(a-d) / d} x_{d}^{-a / d}\right], \quad a=2, \ldots, d .
$$

It follows from [9, Prop. 2] that there exists an open set $\mathscr{V} \subset \mathbb{C}^{d+1} \backslash \Sigma$ of the form

$$
\mathscr{V}:=\left\{x \in \mathbb{C}^{d+1}:\left|x_{0}\right|^{d-j}\left|x_{d}\right|^{j}>M\left|x_{j}\right|^{d} ; j \neq 0, d\right\},
$$

for some positive real constant $M$, where all these series converge, and, according to [22, Theorem 3.2], locally on $\mathscr{V}$ they define the holomorphic $d$ roots of the generic polynomial $\sum_{j=0}^{d} x_{j} \cdot t^{j}$. Given a positive integer $s$ we consider the powers $\rho_{i}^{s}(x)$ and write

$$
\rho_{i}^{s}(x)=\sum_{b=1}^{d} \xi_{i}^{b} \cdot \theta_{b}(x)
$$

where

$$
\theta_{b}(x)=\sum_{a_{1}+\cdots+a_{s}=b+\ell d}(-1)^{\ell} \prod_{j=1}^{s} \sigma_{a_{j}}(x) .
$$

We now consider the $\mathscr{A}$-hypergeometric system associated with the matrix (0.4) and recall that we are assuming that $\operatorname{gcd}\left(k_{1}, \ldots, k_{m}, d\right)=1$. Let $J$ denote the complement of $\left\{0, k_{1}, \ldots, k_{m}, d\right\}$ in $\{0,1, \ldots, d\}$, and $V_{J}$ the $(m+2)$-dimensional subspace of $\mathbb{C}^{d+1}$ obtained by setting $x_{j}=0, j \in J$. Note that $\mathscr{V} \cap V_{J}$ is nonempty.

Lemma 2.5. For $b=1, \ldots, d-1$, the restriction of $\theta_{b}(x)$ to $V_{J}$ is nontrivial. The same is true of any of its derivatives $D_{u} \theta_{b}, u \in \mathbb{N}^{m+2}$, with respect to variables $x_{i}$, $i \notin J$. 
Proof. Note that if $\rho^{s}=\left(\rho_{1}^{s}, \ldots, \rho_{d}^{s}\right)^{T}$ and $\theta=\left(\theta_{1}, \ldots, \theta_{d}\right)^{T}$, then $\theta=M^{-1} \cdot \rho^{s}$ where $M$ is the nonsingular matrix $M=\left(\xi_{i}^{a}\right), i, a=1, \ldots, d$. In particular, $\theta_{b}(x) \in$ $\mathscr{H}((0,-s))(U)$.

We now claim that for any set of indices $a_{1}, \ldots, a_{s}$ such that $a_{1}+\cdots+a_{s}=b+\ell d$, for some $\ell \in \mathbb{N}$,

$$
\theta_{b}(x)=\lambda \cdot\left[x_{a_{1}-1} \cdots x_{a_{s}-1} x_{0}^{(b+(\ell-s) d) / d} x_{d}^{-(b+\ell d) / d}\right],
$$

for some nonzero constant $\lambda$. Indeed, forgetting for the moment the coefficients, suppose that $x^{w}=x^{w^{(1)}} \cdots x^{w^{(s)}}$ is a monomial appearing in the product $\prod_{j=1}^{s} \sigma_{a_{j}^{\prime}}(x)$ where $a_{1}^{\prime}+\cdots+a_{s}^{\prime}=b+\ell^{\prime} d$. Then $\mathscr{A} \cdot w=(0,-s)^{T}$, and, therefore, $x^{w}$ must differ from the monomial inside the bracket (2.12) by a monomial of the form $x^{v}$, with $v \in \mathscr{L}$. This means that all the monomials in the power series of $\theta_{b}(x)$ appear in the $\Gamma$-series of (2.12). But, on the other hand, since we already know that $\theta_{b}(x)$ is $\mathscr{A}$-hypergeometric, if a monomial such as the one in (2.12) appears in its expansion, then the whole $\Gamma$-series must appear with the appropriate coefficients.

Suppose now that we set the variables $x_{j}, j \in J$, equal to zero. We may assume without loss of generality that in the bracket in $(2.12), a_{1}-1, \ldots, a_{r}-1$ are the only indices in $J$. Since $\operatorname{gcd}\left(k_{1}, \ldots, k_{m}, d\right)=1$, there exist positive integers $p_{d}^{\prime}, p_{d}^{\prime \prime}, p_{1}, \ldots, p_{m}$ such that

$$
\left(a_{1}-1\right)+\cdots+\left(a_{r}-1\right)+p_{d}^{\prime} d=p_{1} k_{1}+\cdots+p_{m} k_{m}+p_{d}^{\prime \prime} d .
$$

Setting $p_{d}=p_{d}^{\prime \prime}-p_{d}^{\prime}$, there exists $p_{0} \in \mathbb{Z}$ such that

$$
v:=p_{0} e_{0}+p_{1} e_{k_{1}}+\cdots+p_{m} e_{k_{m}}+p_{d} e_{d}-e_{a_{1}-1}-\cdots-e_{a_{r}-1} \in \mathscr{L} .
$$

Consequently, multiplication of the monomial in the bracket (2.12) by $x^{v}$ yields a term in the $\Gamma$-series that does not involve any variables from the index set $J$. On the other hand, it is easy to check that all coefficients $\gamma\left(u_{i}, v_{i}\right)$ are nonzero, and, therefore, the restriction of $\theta_{b}(x)$ is nontrivial.

The statement about the derivatives of $\theta_{b}(x)$ follows from the fact that for $b<d$, the exponents of $x_{0}$ and $x_{n}$ in the bracket in (2.12) are not integers, while the exponents with which any of the other variables $x_{k_{i}}$ appear in the $\Gamma$-series cannot be bounded, since for any $\ell \in \mathbb{N}$, the element

$$
\ell \cdot\left(d e_{k_{i}}-\left(d-k_{i}\right) e_{0}-k_{i} e_{d}\right) \in \mathscr{L} .
$$

Proof of Theorem 2.4. We consider first the case $\alpha=(0,-s)$, with $s$ a positive integer. Then $\psi_{j}(\alpha)=\rho_{j}^{s} / s$ and, since $\rho^{s}=M \cdot \theta$ with $M$ nonsingular, $\rho_{1}^{s}, \ldots, \rho_{d}^{s}$ are linearly independent if and only if $\theta_{1}(x), \ldots, \theta_{d}(x)$ are so. But comparing the exponents of $x_{d}$ in the corresponding $\Gamma$-series, it is clear that $\theta_{1}(x), \ldots, \theta_{d}(x)$ is linearly independent unless their restriction to $V_{J}$ vanishes. On the other hand, Lemma 2.5 
asserts that only $\theta_{d}(x)$ may be identically zero when restricted to $V_{J}$. Hence, the dimension of the linear span of $\rho_{1}^{s}(x), \ldots, \rho_{d}^{s}(x)$ is at least $d-1$ and is exactly $d-1$ if and only if $\theta_{d}(x)=0$. But,

$$
\sum_{i=1}^{d} \rho_{i}^{s}(x)=\sum_{b=1}^{d}\left(\sum_{i=1}^{d} \xi_{i}^{b}\right) \cdot \theta_{b}(x)=-d \theta_{d}(x) .
$$

Thus, $\theta_{d}(x)=0$ if and only if $\rho_{1}^{s}(x)+\cdots+\rho_{d}^{s}(x)=0$.

The assertion for $\alpha=(0, s)$ with $s$ a positive integer follows from symmetry. In view of the definition (2.6), the statement for $\alpha_{1}<0$ follows from that for $\alpha_{1}=0$, using the assertion in Lemma 2.5 about the derivatives of the $\Gamma$-series $\theta_{b}(x)$.

It remains to consider the case $\alpha_{1}>0$. Suppose there is a nontrivial linear relation $\sum_{i=1}^{d} \lambda_{i} \cdot \psi_{i}(\alpha)=0$; because of (2.7), applying the derivative $\partial^{\alpha_{1}} / \partial x_{0}^{\alpha_{1}}$ we obtain

$$
\sum_{i=1}^{d} \lambda_{i} \cdot \psi_{i}\left(\left(0, \alpha_{2}\right)\right)=0
$$

But $\alpha \notin I(\mathscr{A})$ implies that $\left(0, \alpha_{2}\right) \notin I(\mathscr{A})$, and, therefore, $\lambda_{1}=\cdots=\lambda_{d}$, and the proof is complete.

The following result implies that the holonomic rank of the $\mathscr{A}$-hypergeometric system is at least $d+1$ for all exponents $\alpha \in E(\mathscr{A})$.

Theorem 2.6. For any $\alpha \in E(\mathscr{A}), \operatorname{dim}\left(\mathscr{H}_{\mathrm{alg}}(\alpha)(\mathcal{U})\right) \geq d+1$.

Proof. For any $\alpha \in E(\mathscr{A})$ the $\mathscr{A}$-hypergeometric Laurent polynomials $\Psi_{0}^{\mathscr{A}}(x)$ and $\Psi_{d}^{A}(x)$ are both nontrivial. In particular, $0<\alpha_{2}<d \alpha_{1}$. From (i) in Proposition 2.3, we have that

$$
\psi_{1}(\alpha)+\cdots+\psi_{d}(\alpha) \neq 0
$$

and Theorem 2.4 implies that $\psi_{1}(\alpha), \ldots, \psi_{d}(\alpha)$ are linearly independent. Moreover, we show next that so are the functions $\psi_{1}(\alpha), \ldots, \psi_{d}(\alpha), \Psi_{0}^{A A}(\alpha)$. Suppose there is a nontrivial linear combination $\Psi_{0}^{A}(\alpha)=\sum_{i=1}^{d} \lambda_{i} \cdot \psi_{i}(\alpha), \lambda_{i} \in \mathbb{C}$. By differentiation we obtain a similar relation

$$
\Psi_{0}^{A}\left(\left(0, \alpha_{2}\right)\right)=\frac{\partial^{\alpha_{1}} \Psi_{0}^{A}(\alpha)}{\partial x_{0}^{\alpha_{1}}}=\sum_{i=1}^{d} \lambda_{i} \cdot \frac{\partial^{\alpha_{1}} \psi_{i}(\alpha)}{\partial x_{0}^{\alpha_{1}}}=\sum_{i=1}^{d} \lambda_{i} \cdot \psi_{i}\left(\left(0, \alpha_{2}\right)\right) .
$$

But (1.10) implies that $\Psi_{d}^{A}\left(\left(0, \alpha_{2}\right)\right)=0$, and, therefore, $\Psi_{0}^{A}\left(\left(0, \alpha_{2}\right)\right)=\sum_{i=1}^{d}$ $\psi_{i}\left(\left(0, \alpha_{2}\right)\right)$. Since according to (ii) in Proposition $1.14 \Psi_{0}^{\mathcal{A}}\left(\left(0, \alpha_{2}\right)\right) \neq 0$, we have that all $\lambda_{i}=1$. This implies that $\Psi_{0}^{A}(\alpha)=\sum_{i=1}^{d} \psi_{i}(\alpha)=\Psi_{0}^{A}(\alpha)+\Psi_{d}^{A}(\alpha)$; that is, $\Psi_{d}^{A}(\alpha)=0$, which contradicts our assumption.

Corollary 2.7. The toric ring $R=\mathbb{C}\left[\xi_{0}, \ldots, \xi_{d}\right] / \mathscr{I}_{\mathscr{A}}$ is Cohen-Macaulay if and only if, for every $\alpha \in \mathbb{C}^{2}$, the dimension of the space of A-hypergeometric functions of exponent $\alpha$, at a generic point, is equal to $d$. 
Proof. The "only if" direction is Theorem 2 in [9] (see also [10] and [2]). To prove the converse we note that, because of Proposition 1.6, if $R$ is not Cohen-Macaulay, then $E(\mathscr{A}) \neq \emptyset$, and the result follows from Theorem 2.6.

3. Bases of solutions and holonomic rank. In this section we determine the holonomic rank of the $\mathscr{A}$-hypergeometric system associated with a monomial curve for all integral exponents and exhibit explicit bases of local solutions constructed in terms of the roots of the generic polynomial (0.5).

Four different scenarios need to be considered.

- The exponent $\alpha \in I(\mathscr{A})$ : In this case $r(\alpha)=d$ and we construct, in Theorem 3.1, $d-1$ local solutions which, together with the hypergeometric polynomial $\Phi^{\mathscr{A}}(\alpha)$, define a basis of solutions.

- $\alpha \in E(\mathscr{A})$ : We now have $r(\alpha)=d+1$ and we have constructed $d+1$ (algebraic) local solutions in Theorem 2.6.

- $\alpha \in\left(E_{0}(\mathscr{A}) \cup E_{d}(\mathscr{A})\right) \backslash E(\mathscr{A})$ : The holonomic rank equals $d$ and we have from Theorem 2.4 a basis of algebraic solutions.

- $\alpha \in J(\mathscr{A})$ : Then $\mathscr{R}(\alpha)=\{0\}, r(\alpha)=d$, and we construct a basis of local solutions in Theorem 3.5.

In Theorem 3.7 we determine the holonomic rank $r(\alpha)$ for all $\alpha \in \mathbb{Z}^{2}$. Our starting point is a result of Adolphson [2, Cor. 5.20], which states that even without assuming that the ring $R$ is Cohen-Macaulay, the equality $r(\alpha)=\operatorname{vol}(P)$ holds for so-called semi-nonresonant exponents $\alpha$. In our particular case, this condition is equivalent to $\alpha$ being in the Euler-Jacobi cone (1.13).

We consider first the case when $\alpha \in I(\mathscr{A})$, that is, when the $\mathscr{A}$-hypergeometric polynomial $\Phi^{A}(\alpha ; x) \neq 0$. By Proposition 2.1, given a root $\rho(x)$, the function $\psi_{\rho}(\alpha ; x)$ satisfies the higher-order equations (0.1) but, clearly, not the homogeneity equations (0.2). However, if we fix a choice of a root $\hat{\rho}$, then, for any other root $\rho$, the function

$$
\tau_{\rho}(\alpha):=\sum_{\substack{i=0 \\ i \neq \alpha_{2}}}^{d \alpha_{1}} \Phi^{\mathscr{A}}\left(\alpha_{1}, i\right) \frac{\rho^{i-\alpha_{2}}-\hat{\rho}^{i-\alpha_{2}}}{i-\alpha_{2}}+\Phi^{\mathscr{A}}(\alpha) \log \left(\frac{\rho}{\hat{\rho}}\right)
$$

is $\mathscr{A}$-hypergeometric with exponent $\alpha$. Indeed, it differs from $\psi_{\rho}(\alpha)-\psi_{\hat{\rho}}(\alpha)$ by a constant multiple of $\Phi^{\mathscr{A}}(\alpha)$.

THEOREM 3.1. Given $\alpha \in I(\mathscr{A})$ and a choice of a root $\hat{\rho}$ of $f(x ; t)$ on $U$, the functions $\Phi^{\mathscr{A}}(\alpha)$ and $\tau_{\rho}(\alpha)$, where $\rho$ runs over all roots of $f(x ; t)$ on $\mathcal{U}$ different from $\hat{\rho}$, are linearly independent, A-hypergeometric functions.

Proof. Suppose $\alpha=\mathscr{A} \cdot u, u \in \mathbb{N}^{m+2}$, and suppose there is a nontrivial linear combination

$$
\lambda \Phi^{\mathscr{A}}(\alpha)+\sum_{\rho \neq \hat{\rho}} \lambda_{\rho} \tau_{\rho}(\alpha)=0 .
$$


Applying the operator $D_{u}$ and using (1.2) and (2.7), we may assume that $\alpha=(0,0)$ and, consequently, $\Phi^{A}(\alpha)=1, \tau_{\rho}(\alpha)=\log (\rho / \hat{\rho})$, and

$$
\lambda+\sum_{\rho \neq \hat{\rho}} \lambda_{\rho} \log \left(\frac{\rho}{\hat{\rho}}\right)=0 .
$$

Implicit differentiation of the equation $f(x ; \rho(x))=0$ yields $\partial \rho / \partial x_{\ell}=-\rho^{\ell} / f^{\prime}(\rho)$, $\ell \geq 0$ and, consequently,

$$
\frac{\partial \log \rho}{\partial x_{d}}=\frac{1}{d} \frac{\partial \rho^{d}}{\partial x_{0}}=\psi_{\rho}((-1,-d)) .
$$

Hence, derivating (3.2), we obtain

$$
\sum_{\rho \neq \hat{\rho}} \lambda_{\rho}\left(\psi_{\rho}((-1,-d))-\psi_{\hat{\rho}}((-1,-d))\right)=0,
$$

which, in view of Theorem 2.4, implies $\lambda_{\rho}=0$ for all $\rho \neq \hat{\rho}$.

Suppose now that $\alpha \in J(\mathscr{A})$. In particular, $\alpha \notin I(\mathscr{A})$, and, by (ii) in Proposition 2.3, $\Psi^{A}(\alpha)=0$. Recall also that this case includes all integral exponents lying in the Euler-Jacobi cone.

As before, we let $U$ denote a simply connected open set in $\mathbb{C}^{m+2} \backslash \Sigma$, and let $\rho_{1}, \ldots, \rho_{d}$ denote the roots of $f(x ; t)$, for $x \in \mathcal{U}$. Given $\alpha \in \mathbb{Z}^{2}$ such that $\alpha_{1} \geq 0$, we define

$$
\chi(\alpha):=\sum_{j=1}^{d} \psi_{j}(\alpha) \log \left(\rho_{j}\right)
$$

Proposition 3.2. Suppose $\alpha \in J(\mathscr{A})$ is such that $\alpha_{1} \geq 0$. Then the function $\chi(\alpha)$ is $A$-hypergeometric with exponent $\alpha$.

Proof. Since $\Psi^{\mathscr{A}}(\alpha)=\sum_{j=1}^{d} \psi_{j}(\alpha)=0$, it follows that $\chi(\alpha)$ satisfies the equations (0.2) with exponent $\alpha$. In order to check that the higher-order equations (0.1) are satisfied as well, we show, first of all, that if $\alpha_{1}>0$, then

$$
\frac{\partial \chi(\alpha)}{\partial x_{\ell}}=\chi\left(\alpha-\mathscr{A} \cdot e_{\ell}\right) \text {. }
$$

Indeed,

$$
\begin{aligned}
\frac{\partial \chi(\alpha)}{\partial x_{\ell}} & =\sum_{j=1}^{d} \psi_{j}\left(\alpha-\mathscr{A} \cdot e_{\ell}\right) \log \left(\rho_{j}\right)+\sum_{j=1}^{d} \psi_{j}(\alpha) \rho_{j}^{-1} \frac{\partial \rho_{j}}{\partial x_{\ell}} \\
& =\chi\left(\alpha-\mathscr{A} \cdot e_{\ell}\right)+\sum_{i=0}^{d \alpha_{1}} \frac{\Phi^{\mathscr{A}}\left(\left(\alpha_{1}, i\right)\right)}{i-\alpha_{2}}\left(\sum_{j=1}^{d} \rho_{j}^{i-\alpha_{2}-1} \frac{\partial \rho_{j}}{\partial x_{\ell}}\right) .
\end{aligned}
$$


We claim that the second summand is identically zero. In fact,

$$
\sum_{j=1}^{d} \rho_{j}^{i-\alpha_{2}-1} \frac{\partial \rho_{j}}{\partial x_{\ell}}=\frac{\partial}{\partial x_{\ell}}\left(\sum_{j=1}^{d} \frac{\rho_{j}^{i-\alpha_{2}}}{i-\alpha_{2}}\right) .
$$

Assume $\Phi^{\mathscr{A}}\left(\alpha_{1}, i\right) \neq 0$, that is, there exists $w \in \mathbb{N}^{m+2}$ such that $\mathscr{A} \cdot w=\left(\alpha_{1}, i\right)$. If $\sum_{j=1}^{d} \rho_{j}^{i-\alpha_{2}}$ does not vanish identically, there exists $v \in \mathbb{Z}^{m+2}$ such that $v_{k_{i}} \geq 0$ for all $i=1, \ldots, m$, and either $v_{0} \geq 0$ or $v_{d} \geq 0$, verifying $\mathscr{A} \cdot v=\left(0, \alpha_{2}-i\right)$. But then $\mathscr{A} \cdot(v+w)=\alpha$, which contradicts the fact that $\Psi^{\mathscr{A}}(\alpha)=0$. This proves (3.4).

Arguing as in the proof of Proposition 2.1, the proof of Proposition 3.2 now reduces to the following.

Lemma 3.3. Given $s \in \mathbb{Z}$, $s \neq 0$, and $u \in \mathbb{N}^{m+2}$, let $\beta=\mathscr{A} \cdot u$ and $\gamma=(0,-s)-\beta$. Then, if $\Psi^{A}(\gamma)=0$, the derivative

$$
D_{u}\left(\sum_{j=1}^{d} \frac{\rho_{j}^{s}}{s} \log \rho_{j}\right)
$$

depends only on $\gamma$.

Proof. We argue as in (2.5); locally on $x$,

$$
\begin{aligned}
2 \pi i D_{u}\left(\sum_{j=1}^{d} \frac{\rho_{j}^{s}}{s} \log \rho_{j}\right) & =\sum_{j=1}^{d} \int_{\Gamma_{j}} \frac{t^{s}}{s} \log t D_{u}\left(\frac{f^{\prime}(x ; t)}{f(x ; t)}\right) d t \\
& =-\sum_{j=1}^{d} \int_{\Gamma_{j}} \frac{d}{d t}\left(\frac{t^{s}}{s} \log t\right) D_{u}(\log f(x ; t)) d t \\
& =c \cdot\left[\sum_{j=1}^{d} \int_{\Gamma_{j}} \log t \frac{t^{\beta_{2}+s-1}}{f^{\beta_{1}}} d t+\sum_{j=1}^{d} \int_{\Gamma_{j}} \frac{t^{s-1}}{s} \frac{t^{\beta_{2}}}{f^{\beta_{1}}} d t\right],
\end{aligned}
$$

where $\Gamma_{j}$ is a sufficiently small loop in the complex plane enclosing only the root $\rho_{j}$ and $c=(-1)^{\beta_{1}-1}\left(\beta_{1}-1\right)$ !. Now, according to (2.5), the last sum in (3.5) agrees, up to constant, with

$$
\sum_{j=1}^{d} D_{u}\left(\frac{\rho_{j}^{s}}{s}\right)=\sum_{j=1}^{d} D_{u}\left(\psi_{j}((0,-s))\right)=\sum_{j=1}^{d} \psi_{j}(\gamma)=\Psi^{\mathscr{A}}(\gamma)=0 .
$$

Hence, the lemma and Proposition 3.2 follow.

We now extend the definition (3.3) of $\chi(\alpha)$ to the case $\alpha \in \mathbb{Z}^{2}, \alpha_{1}<0$, by setting 


$$
\chi(\alpha)= \begin{cases}D_{0}^{-\alpha_{1}} \chi\left(\left(0, \alpha_{2}\right)\right), & \text { if } \alpha_{2} \neq 0 \\ D_{k_{1}} D_{0}^{-\alpha_{1}-1} \chi\left(\left(0, k_{1}\right)\right), & \text { if } \alpha_{2}=0,\end{cases}
$$

where we recall

$$
\chi((0, s))=\sum_{j=1}^{d} \frac{\rho_{j}^{-s}}{-s} \log \rho_{j}, \quad s \neq 0 .
$$

Proposition 3.4. Let $\alpha \in \mathbb{Z}^{2}, \alpha_{1}<0$. Then

(i) $\chi(\alpha)(t * x)=t^{\alpha_{2}} \chi(\alpha)(x)-t^{\alpha_{2}} \log t \Psi^{\mathscr{A}}(\alpha ; x)$, where $t \in \mathbb{C}^{*}$, and

$$
t * x=\left(x_{0}, t^{k_{1}} x_{k_{1}}, \ldots, t^{d} x_{d}\right)
$$

(ii) if $\alpha \in J(\mathscr{A}), \chi(\alpha)$ is $\mathscr{A}$-hypergeometric with exponent $\alpha$;

(iii) for $M$ sufficiently large and $j=1, \ldots, m$,

$$
D_{0}^{M\left(d-k_{j}\right)} D_{d}^{M k_{j}}(\chi(\alpha))=D_{k_{j}}^{M d}(\chi(\alpha)) .
$$

Proof. In view of (3.7), the first assertion follows from (3.6), together with the fact that $\rho_{j}(t * x)=t^{-1} \rho_{j}(x), j=1, \ldots, d$.

If $\alpha \in J(\mathscr{A}), \Psi^{\mathscr{A}}(\alpha ; x)=0$, and it follows from (i) that $\chi(\alpha)$ satisfies the homogeneity equations (0.2). On the other hand, if $\alpha \in J(\mathscr{A})$, the same is true of $\alpha-\mathscr{A} \cdot u$ for every $u \in \mathbb{N}^{m+2}$. Hence, it follows from Lemma 3.3 that $\chi(\alpha)$ satisfies the equations $(0.1)$.

The last assertion follows again from Lemma 3.3 for $M$ so that $\alpha-\left(M d, M d k_{j}\right) \in$ $\mathscr{E} \mathscr{J} \subset J(\mathscr{A})$.

Theorem 3.5. Let $\alpha \in J(\mathscr{A})$. Let $\mathcal{U}, \rho_{1}, \ldots, \rho_{d}$, be as above. Then the functions $\psi_{1}(\alpha), \ldots, \psi_{d-1}(\alpha), \chi(\alpha) \in \mathscr{H}(\alpha)(U)$ are linearly independent.

Proof. In view of Theorem 2.4, it is enough to show that $\chi(\alpha)$ is not an algebraic function. In fact, we show that its orbit under the monodromy action of $\pi_{1}\left(\mathbb{C}^{m+2} \backslash \Sigma\right)$ is infinite.

For generic values $a_{k_{1}}, \ldots, a_{d}$, the polynomial $f\left(\left(0, a_{k_{1}}, \ldots, a_{d}\right) ; t\right)$ has a root of multiplicity $k_{1}$ at the origin and $d-k_{1}$ simple, nonzero roots. Thus, for $\left|a_{0}\right|$ small, relative to $\left|a_{k_{1}}\right|, \ldots,\left|a_{d}\right|$, the polynomial $f(a ; t)$ has simple roots, and $k_{1}$ of them, say, $\rho_{1}, \ldots, \rho_{k_{1}}$, are very close to the $k_{1}$ th roots of $-a_{0} / a_{k_{1}}$. This means that analytic continuation of the roots along the loop

$$
\gamma(\theta):=\left(\exp \left(2 \pi i k_{1} \theta\right) a_{0}, a_{k_{1}}, \ldots, a_{d}\right), \quad \theta \in[0,1]
$$

returns the roots to their original values; but, for any choice of logarithm for all roots of $f$ near $a$,

$$
\gamma^{*}\left(\log \left(\rho_{j}\right)\right)= \begin{cases}\log \left(\rho_{j}\right)+2 \pi i, & \text { if } j=1, \ldots, k_{1} \\ \log \left(\rho_{j}\right), & \text { otherwise. }\end{cases}
$$


Since $\alpha \notin I(\mathscr{A})$, it follows from (2.1) and (2.6) that $\gamma^{*}\left(\psi_{j}(\alpha)\right)=\psi_{j}(\alpha)$ for any $j=1, \ldots, d$. Therefore, for $\alpha_{1} \geq 0$,

$$
\gamma^{*}(\chi(\alpha))=\gamma^{*}\left(\sum_{j=1}^{d} \psi_{j}(\alpha) \log \left(\rho_{j}\right)\right)=\chi(\alpha)+2 \pi i \sum_{j=1}^{k_{1}} \psi_{j} .
$$

Since $0<k_{1}<d$, by Theorem $2.4, \psi_{1}+\cdots+\psi_{k_{1}} \neq 0$, and, therefore, the orbit of $\chi(\alpha)$ under successive powers of $\gamma$ is infinite.

Suppose now that $\alpha_{1}<0$. Then,

$$
\chi(\alpha)=\frac{\partial^{-\alpha_{1}}}{\partial x_{0}^{-\alpha_{1}}}\left(\sum_{j=1}^{d} \psi_{j}\left(\left(0, \alpha_{2}\right)\right) \log \rho_{j}\right)=\sum_{j=1}^{d} \psi_{j}(\alpha) \log \rho_{j}+R(\alpha) .
$$

It is straightforward to check that $R(\alpha)$ is algebraic and invariant under the monodromy action; that is, $R(\alpha)$ is a rational function. Since we have just shown that the function $\sum_{j=1}^{d} \psi_{j}(\alpha) \log \rho_{j}$ is not algebraic, the proof is complete.

3.6. Remark. Note that since the function $\chi(\alpha)$ is not algebraic, any rational $\mathscr{A}$ hypergeometric function $R$ with exponent $\alpha$ in the Euler-Jacobi cone must be a linear combination of $\psi_{1}(\alpha), \ldots, \psi_{d}(\alpha)$. On the other hand, with arguments similar to those in the proof of Theorem 3.5, it is possible to show the existence of a loop $\gamma$ whose action on the roots is a cyclic permutation. It is then easy to see that $R$ must be a constant multiple of $\sum_{j=1}^{d} \psi_{j}(\alpha)$, and, therefore, it must vanish. This gives an alternative proof of Theorem 1.9.

TheOREM 3.7. For every $\alpha \in \mathbb{Z}^{2}$,

$$
d \leq r(\alpha) \leq d+1
$$

Moreover, $r(\alpha)=d+1$ if and only if $\alpha \in E(\mathscr{A})$.

Proof. Note first that the lower bound follows from Theorem 2.4 (for $\alpha \in E_{0}(\mathscr{A}) \cup$ $E_{d}(\mathscr{A})$ ), Theorem 3.1 (for $\alpha \in I(\mathscr{A})$ ), and Theorem 3.5 (for $\alpha \in J(\mathscr{A})$ ).

Suppose now that $\alpha$ is in the Euler-Jacobi cone $\mathscr{E} \mathscr{E}$. Then, as we observed before, $\alpha$ is semi-nonresonant in the sense of Adolphson, and it follows from [2, Cor. 5.20] that $r(\alpha)=d$. For any $\alpha \in \mathbb{Z}^{2}$, there exist $u \in \mathbb{N}^{m+2}$ such that $\alpha-\mathscr{A} \cdot u$ lies in $\mathscr{E} \mathscr{g}$, and, because of Theorem 1.9, for any such $u$, the kernel of the linear map

$$
D_{u}: \mathscr{H}(\alpha) \rightarrow \mathscr{H}(\alpha-\mathscr{A} \cdot u)
$$

contains $\mathscr{R}(\alpha)$. We determine the dimension of $\mathscr{H}(\alpha)$ by computing the kernel and the image of $D_{u}$ for suitable $u$.

Suppose first that $\alpha \in J(\mathscr{A})$. For $u=\ell e_{k_{1}}, \ell \gg 0$, we have $\alpha-\mathscr{A} \cdot u \in \mathscr{E} \mathscr{g}$, 
and it follows from Proposition 1.2 and Corollary 1.5 that $\operatorname{ker}\left(D_{u}\right)=\mathscr{R}(\alpha)=\{0\}$. Therefore, $D_{u}$ is a monomorphism, which implies that $\operatorname{dim} \mathscr{H}(\alpha) \leq d$. Since it is at least $d$, we deduce that $\operatorname{dim} \mathscr{H}(\alpha)=d$. Suppose now that $\alpha \notin J(\mathscr{A})$; then $\operatorname{dim}(\mathscr{R}(\alpha))=1$ or 2 . We can again choose $u=\ell e_{k_{1}}, \ell \gg 0$, so that $\beta:=\alpha-\mathscr{A} \cdot u \in$ $\mathscr{E} \mathscr{E}$. As the kernel of $D_{u}$ is precisely $\mathscr{R}(\alpha)$, it is enough to show that, for some $\ell$ sufficiently large, the dimension of the image of $D_{u}$ is $d-1$.

From (2.6) and Theorem 3.5, we deduce that the functions $\psi_{j}(\beta)$ generate a subspace of the image of dimension at least $d-1$. The proof is completed by showing that the function $\chi(\beta)$ defined in (3.6) is not in the image $D_{u}(\mathscr{H}(\alpha))$.

Consider first the case $\alpha \in E_{0}(\mathscr{A})$. Choosing $\ell=s d$, we factor $D_{u}=D_{k_{1}}^{s d}=$ $D_{d}^{k_{1} s} D_{0}^{\left(d-k_{1}\right) s}$. Set $\alpha^{\prime}:=\alpha-\left(\left(d-k_{1}\right) s, 0\right)$. It is enough to show that $\chi(\beta) \notin D_{d}^{k_{1} s}$ $\left(\mathscr{H}\left(\alpha^{\prime}\right)\right)$. Note that $\alpha^{\prime} \in E_{0}(\mathscr{A})$ as well, and, therefore, we may assume without loss of generality that $\alpha_{1}<0$ and $\beta=\alpha-\ell e_{d} \in \mathscr{E} \mathscr{E}$ for some sufficiently big $\ell$.

Let $\chi(\alpha)$ be as in (3.6). Since $\beta \in \mathscr{E} \mathscr{g}$, it follows from Lemma 3.3 that $D_{d}^{\ell}(\chi(\alpha))=$ $\chi(\beta)$. Therefore, if $\phi \in \mathscr{H}(\alpha)$ is such that $D_{d}^{\ell}(\phi)=\chi(\beta)$, we must have

$$
\phi=\chi(\alpha)+F,
$$

where $F$ depends polynomially on $x_{d}$. On the other hand, because of (iii) in Proposition 3.4 and the fact that $\phi$ is hypergeometric, we have

$$
D_{k_{j}}^{M d}(F)=D_{0}^{M\left(d-k_{j}\right)} D_{d}^{M k_{j}}(F)
$$

for all $M$ large enough. This implies that $F$ is polynomial on $x_{k_{1}}, \ldots, x_{k_{m}}$ as well.

But, it follows from (i) in Proposition 3.4 that

$$
F(t * x)=t^{\alpha_{2}} F(x)+t^{\alpha_{2}} \log t \Psi(\alpha)(x),
$$

which is impossible, since the fact that the action of $t$ does not affect $x_{0}$ implies that $F(t * x)$ is polynomial in $t$.

By symmetry, the result also holds for $\alpha \in E_{d}(\mathscr{A})$. Thus, it remains to consider the case $\alpha \in I(\mathscr{A})$. For $\ell$ large enough, so that $\alpha_{1}^{\prime}=\alpha_{1}-\ell\left(d-k_{1}\right)<0$, we have $\alpha^{\prime}=\left(\alpha_{1}^{\prime}, \alpha_{2}\right) \in E_{0}(\mathscr{A})$, and an argument similar to the one above yields the result.

3.8. Remark. In [19, Thm. 12.2], M. Saito, B. Sturmfels, and N. Takayama prove Theorem 3.7 by the method of Gröbner deformations. They also show that the lower bound $\operatorname{vol}(P) \leq r(\alpha)$ holds for arbitrary $\mathscr{A}[19$, Theorem 11.1].

Given $\alpha \in \mathbb{Z}^{2}$, define $\hat{\mathscr{H}}(\alpha):=\mathscr{H}(\alpha) / \mathscr{R}(\alpha)$ if $\mathscr{R}(\alpha) \neq\{0\}$ and $\hat{\mathscr{H}}(\alpha):=$ $\mathscr{H}(\alpha) / \mathbb{C} \cdot \chi(\alpha)$ if $\mathscr{R}(\alpha)=\{0\}$.

Corollary 3.9. For every $\alpha \in \mathbb{Z}^{2}$,

(i) $\operatorname{dim}(\hat{\mathscr{H}}(\alpha))=d-1$; 
(ii) For every $u \in \mathbb{N}^{m+2}$, the operator

$$
D_{u}: \hat{\mathscr{H}}(\alpha) \rightarrow \hat{\mathscr{H}}(\alpha-\mathscr{A} \cdot u)
$$

is an isomorphism.

\section{REFERENCES}

[1] S. Abramov and K. Kvasenko, "Fast algorithms to search for the rational solutions of linear differential equations with polynomial coefficients" in Proceedings of the 1991 International Symposium on Symbolic and Algebraic Computation: ISSAC '91 (Bonn, Germany, 1991), ed. by S. M. Watt, ACM Press, New York, 1991, 267-270.

[2] A. Adolphson, Hypergeometric functions and rings generated by monomials, Duke Math. J. 73 (1994), 269-290.

[3] A. C. Avram, E. Derrick, and D. Jančŕć, On semi-periods, Nuclear Phys. B 471 (1996), 293-308.

[4] V. BATYREV, Variations of the mixed Hodge structure of affine hypersurfaces in algebraic tori, Duke Math. J. 69 (1993), 349-409.

[5] V. BATYREV AND D. van STRATEN, Generalized hypergeometric functions and rational curves on Calabi-Yau complete intersections in toric varieties, Comm. Math. Phys. 168 (1995), 493-533.

[6] W. Bruns and J. Herzog, Cohen-Macaulay Rings, Cambridge Stud. Adv. Math. 39, Cambridge Univ. Press, Cambridge, 1993.

[7] I. M. Gel'Fand, M. Kapranov, and A. Zelevinsky, Generalized Euler integrals and Ahypergeometric functions, Adv. Math. 84 (1990), 255-271.

[8] - Discriminants, Resultants, and Multidimensional Determinants, Math. Theory Appl., Birkhäuser, Boston, 1994.

[9] I. M. Gel'Fand, A. Zelevinsky, and M. Kapranov, Hypergeometric functions and toral manifolds, Funct. Anal. Appl. 23 (1989), 94-106.

[10] - Correction to "Hypergeometric functions and toral manifolds," Funct. Anal. Appl. 27 (1994), 295.

[11] _ A-discriminants and Cayley-Koszul complexes, Soviet Math. Dokl. 40 (1990), 239243.

[12] S. Goto, N. Suzuki, And K. Watanabe, On affine semigroup rings, Japan. J. Math. (N.S.) 2 (1976), 1-12.

[13] S. Hosono, "GKZ systems, Gröbner fans, and moduli spaces of Calabi-Yau hypersurfaces" in Topological Field Theory, Primitive Forms, and Related Topics (Kyoto, 1996), Progr. Math. 160, Birkhäuser, Boston, 1998, 239-265.

[14] S. Hosono, B. H. Lian, AND S.-T. YAU, GKZ-generalized hypergeometric systems in mirror symmetry of Calabi-Yau hypersurfaces, Comm. Math. Phys. 182 (1996), 535-577.

[15] - Maximal degeneracy points of GKZ systems, J. Amer. Math. Soc. 10 (1997), 427-443.

[16] A. KhovanskiI, Newton polyhedra and the Euler-Jacobi formula, Russian Math. Surveys 33, no. 6 (1978), 237-238.

[17] K. MAYR, Über die Lösung algebraischer Gleichungssysteme durch hypergeometrische Funktionen, Monatsh. Math. Phys. 45 (1937), 280-313, 435.

[18] M. Saito, B. Sturmfels, and N. Takayama, Hypergeometric polynomials and integer programming, Compositio Math. 155 (1999), 185-204.

[19] - Gröbner deformations of hypergeometric differential equations, to appear in Algorithms Comput. Math., Springer-Verlag, Berlin.

[20] M. F. SINGER, Liouvillian solutions of nth order homogeneous linear differential equations, 
Amer. J. Math. 103 (1981), 661-682.

[21] J. STIEnSTRA, Resonant hypergeometric systems and mirror symmetry, preprint, http://xxx.lanl.gov/abs/alg-geom/9711002.

[22] B. Sturmfels, Solving algebraic equations in terms of A-hypergeometric series, to appear in Discrete Math.

[23] B. Sturmfels and N. TAKayama, "Gröbner bases and hypergeometric functions" in Gröbner Bases and Applications, ed. by B. Buchberger and F. Winkler, London Math. Soc. Lecture Note Ser. 251, Cambridge Univ. Press, New York, 1998, 246-258.

[24] N. Tакауама, Kan: A system for computation in algebraic analysis, 1991, http://www.math.s.kobe-u.ac.jp/KAN/.

[25] N. V. Trung And L. T. HoA, Affine semigroups and Cohen-Macaulay rings generated by monomials, Trans. Amer. Math. Soc. 298 (1986), 145-167.

Cattani: Department of Mathematics and Statistics, University of Massachusetts, AMHERST, MassaCHUSETTS 01003, USA; cattani@math.umass.edu

D’Andrea: Departamento de Matemática, Universidad de Buenos Aires, Ciudad Universitaria, Pabellón I, (1428) Buenos Aires, Argentina; cdandrea@dm.uba.ar

Dickenstein: Departamento de Matemática, Universidad de Buenos Aires, Ciudad Universitaria, Pabellón I, (1428) Buenos Aires, Argentina; alidick@dm.uba.ar 